University of Nebraska - Lincoln

DigitalCommons@University of Nebraska - Lincoln

$2-29-2008$

\title{
Intercomparison and sensitivity analysis of Leaf Area Index retrievals from LAI-2000, AccuPAR, and digital hemispherical photography over croplands
}

S. Garrigues

University of Maryland - College Park, Sebastien.garrigues@gsfc.nasa.gov

N. V. Shabanov

Boston University

K. Swanson

Boston University

J. T. Morisette

NASA Goddard Space Flight Center,

F Baret

INRA

See next page for additional authors

Follow this and additional works at: https://digitalcommons.unl.edu/nasapub

Part of the Physical Sciences and Mathematics Commons

Garrigues, S.; Shabanov, N. V.; Swanson, K.; Morisette, J. T.; Baret, F; and Myneni, R. B., "Intercomparison and sensitivity analysis of Leaf Area Index retrievals from LAI-2000, AccuPAR, and digital hemispherical photography over croplands" (2008). NASA Publications. 16.

https://digitalcommons.unl.edu/nasapub/16

This Article is brought to you for free and open access by the National Aeronautics and Space Administration at DigitalCommons@University of Nebraska - Lincoln. It has been accepted for inclusion in NASA Publications by an authorized administrator of DigitalCommons@University of Nebraska - Lincoln. 


\section{Authors}

S. Garrigues, N. V. Shabanov, K. Swanson, J. T. Morisette, F Baret, and R. B. Myneni 


\title{
Intercomparison and sensitivity analysis of Leaf Area Index retrievals from LAI-2000, AccuPAR, and digital hemispherical photography over croplands
}

\author{
S. Garrigues ${ }^{a, *}$, N.V. Shabanov ${ }^{b}$, K. Swanson ${ }^{b}$, J.T. Morisette ${ }^{c}$, F. Baret ${ }^{d}$, R.B. Myneni ${ }^{b}$ \\ ${ }^{a}$ University of Maryland's Earth System Science Interdisciplinary Center, College Park, MD 20742, USA \\ ${ }^{\mathrm{b}}$ Department of Geography, Boston University, Boston, MA 02215, USA \\ ' NASA Goddard Space Flight Center, Code 614.5, Greenbelt, MD 20771, USA \\ dINRA, UMR1114 (EMMAH), F-84914 Avignon, France
}

\section{A R T I C L E I N F O}

\section{Article history:}

Received 24 May 2007

Received in revised form

23 October 2007

Accepted 29 February 2008

\section{Keywords:}

Effective Plant Area Index

Gap fraction

Optical techniques

LAI-2000

AccuPAR

Digital Hemispherical Photograph

LAI validation

\begin{abstract}
A B S T R A C T
Validation of Leaf Area Index (LAI) derived from moderate resolution remote sensing observations generally involves optical technique to measure ground LAI. As the current validation datasets are derived using multiple optical retrieval techniques, assessment of the consistency between these techniques is required. In this study the effective Plant Area Index $\left(\mathrm{PAI}_{\text {eff }}\right.$ ) retrievals by three major optical instruments, LAI-2000, AccuPAR, and Digital Hemispherical Photographs (DHPs), were analyzed over 10 crops (soybean, corn, alfalfa, sorghum, peanut and pasture) at Manfredi site in Cordoba province, Argentina. The focus of research was on quantifying PAI $_{\text {eff }}$ sensitivity to the type of instrument, retrieval parameters and gap fraction inversion methods as well as environmental conditions (canopy heterogeneity, senescent vegetation, illumination conditions). Results indicate that sensitivity of DHP method to illumination conditions is low (14\% compared to $28 \%$ and $86 \%$ for LAI- 2000 and AccuPAR, respectively). The intercomparison of $\mathrm{PAI}_{\text {eff }}$ retrievals indicates large discrepancies between optical techniques for short canopy over which downward-pointing DHP technique performs better than LAI-2000 and AccuPAR. Better agreement was found for tall canopy without senescent vegetation and low spatial heterogeneity. Overall, discrepancies in PAI $_{\text {eff }}$ between instruments are mainly explained by differences in spatial sampling of transmittance between instruments (over short and heterogeneous canopies) caused by variations in instrument footprint, azimuthal range, and zenith angle spatial resolution (coarser for LAI-2000 than DHP). Our results indicate that DHP is the most robust technique in terms of low sensitivity to illumination conditions, accurate spatial sampling of transmittance, ability to capture gap fraction over short canopy using downward-looking photographs, independence from canopy optical ancillary information, and potential to derive clumping index. It can thus be applied to a large range of canopy structures, and environmental conditions as required by validation protocols.
\end{abstract}

(C) 2008 Elsevier B.V. All rights reserved.

\footnotetext{
* Corresponding author at: NASA GSFC, Mail Code 614.4, Greenbelt, MD 20771, USA. Tel.: +1 3016146646 ; fax: +3016146695.

E-mail address: Sebastien.garrigues@gsfc.nasa.gov (S. Garrigues).
} 


\section{Introduction}

Leaf Area Index (LAI) is a key biophysical variable, used in most global models of climate, ecosystem productivity, biogeochemistry, hydrology, and ecology. It is defined as half the total developed area of green leaves (all sided) per unit ground horizontal surface area (Chen and Black, 1992). Global LAI products are operationally produced from remote sensing observation of major space-borne instruments (e.g. MODIS/ TERRA-AQUA, VEGETATION/SPOT4-5). Assessment of the uncertainties of LAI remote sensing products, i.e., validation of the LAI products with ground measurements, is critical for their proper use in land surface models (Morisette et al., 2006; Garrigues et al., 2008; Weiss et al., 2007). One major shortcoming of the current validation studies is that they poorly document uncertainties associated with LAI measurement-a key information to properly validate satellite product (Garrigues et al., 2008; Weiss et al., 2007).

LAI measurements can be subdivided into two major categories: direct and indirect techniques (Gower et al., 1999; Breda, 2003; Jonckheere et al., 2004). Direct measurements involve destructive harvest techniques and litter fall traps. While laborious, such measurements provide a reference for indirect measurements if the spatial sampling properly represents canopy heterogeneity. The most commonly used indirect techniques in validation studies are optical due to their fast and easy sampling of LAI over large spatial areas (Morisette et al., 2006). Optical techniques are based on measurement of light transmittance through the canopy (Jonckheere et al., 2004; Weiss et al., 2004). They have been implemented with multiple commercial optical instruments, including LAI-2000 Plant Canopy Analyzer (LI-COR, Lincoln, Nebraska USA), AccuPAR (Decagon Devices, Inc., Pullman, Washington, USA), Tracing Radiation and Architecture of Canopies (TRAC, 3rd Wave, Ontario Canada), Digital Hemispherical Photographs (DHPs) among others (Jonckheere et al., 2004).

The reference in situ LAI estimate (called hereafter actual LAI) is achieved using destructive samplings for foliage element area estimates, and locally calibrated allometric relationships to scale these estimates over plots (Chen et al., 1997; Jonckheere et al., 2004). In contrast, optical measurements provide effective LAI, which is an approximation of the actual LAI because of two main issues. First, except when using color DHP, optical measurements do not allow distinguishing between photosynthetically active tissues ("green elements") and other plant elements such as branches, stems, trunks, and senescent leaves, leading to a positive bias in estimated LAI (Chen et al., 1997; Kucharik et al., 1998; Barclay et al., 2000; Stenberg et al., 2003). For this reason, the term Plant Area Index (PAI) will be used in this paper to represent the quantity measured by optical instruments. In addition, most optical techniques retrieve PAI assuming that the spatial distribution of vegetation elements within the canopy is random which is generally not the case in actual canopies (Nilson, 1971; Chen and Black, 1992; Weiss et al., 2004). Typically, the deviation from the random case is quantified through the clumping index, $\Omega$ (Chen and Black, 1992), in the expression $\mathrm{PAI}_{\text {eff }}=\Omega \mathrm{PAI}$, where PAI is the actual PAI as measured from destructive sampling and $\mathrm{PAI}_{\text {eff }}$ is the effective PAI derived from optical measurements assuming a random leaf distribution. Clumping index is equal to 1 for randomly distributed foliage, $>1$ for regularly distributed foliage, and $<1$ for clumped canopies (Weiss et al., 2004). It depends both on plant-scale structure, i.e., the spatial distribution of foliage elements along plant stems and trunks, branches or shoots for trees, and on canopy-scale structure, i.e., the spatial heterogeneity of plant arrangements within the canopy as it occurs in discontinuous canopies (e.g. row crops). When leaf clumping is not accounted for, as in the case of $\mathrm{PAI}_{\text {eff }}$, the actual PAI values may be significantly underestimated (Begue, 1993; Chen and Cihlar, 1995; Stenberg, 1996; Cohen et al., 1997; Fernandes et al., 2003; Jonckheere et al., 2004; Leblanc et al., 2005). Other sources of errors associated with optical measurement includes illumination conditions (direct versus diffuse illumination), variations in the instrument footprint, saturation of the optical signal in dense canopies (gap fraction saturates as LAI approaches to 5-6, Gower et al. (1999)), simplification of leaf optical properties (Leblanc and Chen, 2001; Hyer and Goetz, 2004), poor performances of some instruments (e.g. AccuPAR, LAI-2000) for short canopies, and the ability of the sampling scheme to capture canopy spatial heterogeneity (Weiss et al., 2004).

Some of LAI retrieval errors described above can be corrected through appropriate techniques. Vegetation clumping can be taken into account using clumping index values taken from literature or directly derived from DHP (Van Gardingen et al., 1999; Leblanc et al., 2005) or TRAC (Chen and Cihlar, 1995), while separation of green elements from non-green elements can be achieved with DHP under specific (diffuse) illumination conditions or using near-infrared cameras (Chapman, 2007). Since these corrections are not systematically applied to LAI ground measurements used in current validation datasets (Morisette et al., 2006), evaluating the impact of these uncertainties on LAI retrieval is needed. While destructive sampling is mandatory to assess the absolute accuracy of optical measurements, intercomparison and sensitivity analysis to key retrieval parameters of optical techniques bring useful insights on the relative performances of each instrument (Hyer and Goetz, 2004; Zhang et al., 2005). Besides, since current validation datasets are derived using multiple optical retrieval techniques, assessment of the consistency between these techniques is required. Such exercise is also valuable to identify stable and repeatable measurements of PAI among existing optical retrieval techniques.

In this study, we intercompare $\mathrm{PAI}_{\text {eff }}$ retrievals from LAI2000, AccuPAR, and DHP instruments which are widely used in validation studies. Overall, the literature indicates that consistency between these retrieval techniques vary with vegetation type, range of retrieved LAI, selection of retrieval parameters, and illumination conditions (Martens et al., 1993; Chen et al., 1997; Planchais and Pontailler, 1999; White et al., 2000; Wilhelm et al., 2000; Hyer and Goetz, 2004). Nevertheless, few studies have simultaneously compared these three instruments over the same site, and no consensus between past intercomparison studies has been reached. Up to now most intercomparison of optical instruments have been achieved over forests (Hyer and Goetz, 2004; Zhang et al., 2005), while croplands were underrepresented in such studies.

The objective of this research is to consolidate former experiences on optical measurements, and more specifically 
to investigate the following issues: (1) intercomparison and concurrent sensitivity analysis of $\mathrm{PAI}_{\text {eff }}$ retrievals from LAI2000, AccuPAR and DHP over several types of crop and phenological stages including senescence; (2) exploring the advanced features of DHP measurements, including retrievals of gap fraction from upward- and downward-looking imagery and assessment of vegetation clumping; (3) performing sensitivity analysis of $\mathrm{PAI}_{\text {eff }}$ retrievals to illumination conditions. Since validation field campaigns must be achieved within a short time period centered on satellite acquisition date (Morisette et al., 2006), it is critical to identify retrieval techniques with minimal sensitivity to illumination conditions. (4) Assessing the impact of canopy heterogeneity and scaling methods on $\mathrm{PAI}_{\text {eff }}$ sampled according to typical validation scheme and identifying techniques providing accurate spatial sampling of canopy gaps. This paper is organized as follows. In Section 2, retrieval techniques of each instrument are briefly reviewed. The experimental site and sampling scheme used in this work are described in Section 3. Next (Section 4), results on single sensor sensitivity analysis and sensor intercomparison are presented. Conclusions are drawn in Section 5.

\section{Instruments and retrieval techniques}

\subsection{LAI-2000}

The optics of the LI-COR'S LAI-2000 instrument (LI-COR, 1991) consists of a fisheye lens ( $148^{\circ}$ of field of view) divided into five concentric rings with the following range of zenith angle $\theta$ : 0 $13^{\circ} ; 16-28^{\circ} ; 32-43^{\circ} ; 47-58^{\circ}$ and $61-74^{\circ}$. Each ring simultaneously integrates incoming radiation between 320 and $490 \mathrm{~nm}$ (blue light) over the complete range of azimuth angle. However, in the field, the azimuthal range is restricted to avoid shadowing effects. In this work, the $270^{\circ}$ view cap was used to block off the operator from the instrument's field of view as well as part of influence of the direct light on the sensor (LICOR, 1991 protocol). The retrieval approach implemented in the LAI-2000 software is based on the following assumptions (LI-COR, 1991): (1) foliage elements are absolutely absorbing (black body assumption); (2) foliage elements are randomly distributed without clumping and their azimuthal orientation is uniform; (3) foliage elements are small compared to the area spanned by each ring. Retrievals are based on measurements of canopy transmittance $\tau(\theta)$ for each ring, calculated as the ratio of radiation measurement acquired below and above canopy. Under black body assumption and random leaf distribution, transmittance is equivalent to gap fraction $P(\theta)$, modeled by the Poisson model (Weiss et al., 2004),

$\tau(\theta)=P(\theta)=\exp \left[\frac{-G(\theta, \text { LIDF }) \cdot \text { PAI }_{\mathrm{eff}}}{\cos (\theta)}\right]$

where $G(\theta)$ is the projection function, i.e., the mean projection of a foliage area unit in a plane perpendicular to direction $\theta$, which depends on the Leaf Inclination Distribution Function (LIDF). Several methods can be used to invert Eq. (1) and retrieve $\mathrm{PAI}_{\text {eff }}$ (Weiss et al., 2004).

The standard method implemented in the LAI-2000 software is based on Miller (1967) formula:
$\mathrm{PAI}_{\text {eff }}=-2 \int_{0}^{\pi / 2} \ln (\tau(\theta)) \cos (\theta) \sin (\theta) \mathrm{d} \theta$.

The computation of $\mathrm{PAI}_{\text {eff }}$ from Eq. (2) is achieved by a discrete summation (Welles and Norman, 1991) over the five view zenith angles (center values) of the instrument rings. Several authors have reported that in this method transmittance in the fifth ring is overweighted since it is used in the Eq. (2)'s approximation to estimate transmission in the range $61^{\circ}<\theta<90^{\circ}$ (i.e., $\mathrm{d} \theta=29$ ) while it actually measures radiation in the range $61^{\circ}<\theta<74^{\circ}$ (i.e., $\mathrm{d} \theta=13$ ). This introduces a negative bias of about $8 \%$ as quantified by Leblanc and Chen (2001) who propose to use the actual range of $\theta\left[61^{\circ}, 74^{\circ}\right]$ for the fifth ring. However, in this work we will use the standard LAI-2000 implementation based on the fact that Eq. (2) is valid only if $\theta$ is evaluated in the range $[0, \pi / 2]$.

Retrievals from the standard algorithm will be compared to those derived from two alternative methods which are also implemented in the DHP method described in Section 2.3:

1. Wilson (1963) demonstrated that the G-function can be considered as almost independent of leaf inclination at $\theta$ $\sim 57.5^{\circ}$, namely $G(\theta)$ is constant and always near 0.5 in Eq. (1). In this case $\mathrm{PAI}_{\text {eff }}$ is retrieved from a simple expression derived from Eq. (1),

$\mathrm{PAI}_{\mathrm{eff}} \approx-\frac{\cos \left(\theta_{4}\right)}{0.5} \ln \left(\tau\left(\theta_{4}\right)\right)$,

using the transmittance measured at the fourth ring $\left(\theta_{4}=53^{\circ}\right)$ which is the closest to $\theta=57.5^{\circ}$ (Leblanc and Chen, 2001; Weiss et al., 2004). In the following, this method will be referred as Wilson method.

2. An alternative method consists in using a Look-Up Table technique (Weiss et al., 2004) to invert Eq. (1). To evaluate the G-function, LIDF is assumed to be uniform in azimuth and follow an ellipsoidal distribution for the inclination (Campbell, 1986). In this case, the LIDF is fully characterized with only one parameter, the Average Leaf Inclination Angle (denoted ALIA $_{\text {eff }}$ ). A large number of random combinations of $\mathrm{PAI}_{\text {eff }}\left(0-10\right.$, step 0.01) and ALIA $\mathrm{Aff}_{\text {(10- }}$ $80^{\circ}$, step $2^{\circ}$ ) are used to build a database made of the corresponding gap fraction values simulated in the 5 zenith angles of the LAI-2000 center rings. The inversion process consists in selecting first the 25 LUT elements having the closest RMSE between the simulated and measured gap fractions. Then, the average $\mathrm{PAI}_{\text {eff }}$ and ALIA $_{\text {eff }}$ over the 25 values are chosen as solution. The number of 25 cases was selected in agreement with the uncertainties in gap fraction measurements (Weiss et al., 2004).

The manual for the LAI-2000 recommends utilizing the instrument only under diffuse illumination for optimal performance of hardware (Hyer and Goetz, 2004; Jonckheere et al., 2004).

\subsection{AcCUPAR}

The Decagon's AccuPAR (Decagon Devices Inc, 2001) is a linear quantum sensor comprised of 80 photosensors which mea- 
sure radiation over the PAR (Photosynthetically Active Radiation) wavebands (400-700 nm).

Conversely to LAI-2000, measuring transmittance in a spectral range where leaf reflectance and transmittance are negligible, AccuPAR measures over the PAR region which requires accounting for leaf reflectance and transmittance. Further, AccuPAR retrievals are based on angularly integrated transmittance, as performed by the sensor itself, in contrast to LAI-2000 retrievals, which are based on directional transmittance. As a consequence, information about leaf orientation cannot be estimated from AccuPAR measurements and must be a priori known to retrieve $\mathrm{PAI}_{\mathrm{eff}}$ under direct sunlight condition. The AccuPAR retrieval equation is derived from a simplified formulation of a light transmission model developed by Norman and Jarvis (1975),

$\mathrm{PAI}_{\mathrm{eff}}=\frac{\left[(1-1 / 2 \mathrm{~K}) \cdot f_{\mathrm{b}}-1\right] \ln \tau}{\mathrm{A} \cdot\left(1-0.47 \cdot f_{\mathrm{b}}\right)}$,

In Eq. (4), the parameter $\tau$ is the hemispherically integrated transmittance, computed as the ratio of below-canopy PAR and incident PAR measured by the ceptometer. The term $f_{\mathrm{b}}$ is the beam fraction, i.e., ratio of direct sunlight radiation to total incoming radiation from all ambient sources, as computed by the instrument (cf. AccuPAR manual). Under completely diffuse conditions $f_{\mathrm{b}}=0$ and Eq. (4) simplifies as:

$\mathrm{PAI}_{\mathrm{eff}}=\frac{-\ln \tau}{\mathrm{A}}$.

The term $\mathrm{K}$ in Eq. (4) is the canopy extinction coefficient which incorporates the LIDF. Assuming an ellipsoidal LIDF, it is defined by

$K=\frac{\sqrt{x^{2}+\tan ^{2}\left(\theta_{s}\right)}}{x+1.744(x+1.182)^{-0.733}}$,

where $\theta_{\mathbf{s}}$ is the sun zenith angle and $x$ is the leaf angle distribution parameter defined as the ratio of horizontal to vertical axes of an ellipsoidal LIDF (Campbell, 1986). In the AccuPAR standard mode (used in this work), $x$ is equal to 1 which corresponds to a spherical leaf angle distribution.

The term A in Eq. (4) is a coefficient describing canopy absorptivity and is empirically related to the leaf absorptivity parameter a (equal to 0.9 in AccuPAR standard mode) in the PAR band, as follows,

$\mathrm{A}=0.283+0.785 a-0.159 a^{2}$

The manual for AccuPAR instrument poses no a priori restriction on illumination conditions (Hyer and Goetz, 2004; Jonckheere et al., 2004).

\subsection{DHP}

\subsubsection{Hardware and photography protocol}

DHP technique uses a digital camera with a fisheye lens to measure canopy gap fraction over a wide range of viewing directions (Jonckheere et al., 2004; Weiss et al., 2004; Leblanc et al., 2005). In this work, a Nikon Coolpix990 camera was used at the finest image resolution available $(2048 \times 1536$ pixel matrix recorded in jpeg format), and a FC-E8, Nikon fisheye lens with a field of view of $183^{\circ}$. The camera was calibrated using the method described in WWW $1^{1}$ to compute the actual coordinates of the optical centre of the "camera + fisheye" system.

Photographs were taken in either upward-pointing direction at the ground level for tall canopies (e.g. corn and sorghum) or downward-pointing direction at about $0.8 \mathrm{~m}$ distance from the ground for short canopies (e.g. alfalfa and soybean). The choice of $0.8 \mathrm{~m}$ height is the result of a compromise between a large enough height to properly capture canopy spatial heterogeneity and small enough height to limit the number of mixed pixel during the processing of the images. A bubble level attached to the camera was used to ensure the horizontality of the images. Downward-pointing pictures were acquired with operator facing the sun to avoid operator's shadow in the image. To prevent saturation, the camera was set to automatic exposure conversely to Zhang et al. (2005) who suggest using an overexposure of two stops or more relative to an exposure reference measured for an open sky. While adjusting exposure setting may be required for upward-looking photograph under forest canopy and image processing based on brightness threshold, it is not mandatory for the Can-Eye freeware used here (cf. Section 2.3.2) which analyzes RGB color images possibly taken from above the canopy (less sensitive to exposure settings than upward pictures). Besides, the possibility of masking overexposed area of the image as well as adjusting the image contrast offered by Can-Eye should reduce the effect of camera exposure settings. Regarding illumination conditions, DHP can be used under both direct and diffuse illumination, while literature generally advocates diffuse condition for better performance, particularly for forest (Chen et al., 1991; Frazer et al., 2001; Leblanc et al., 2005). Finally, in order to properly sample the spatial variability within the canopy, a set of $N=13$ photographs were acquired over the same Elementary Sampling Unit (denoted ESU, cf. Section 3) and were simultaneously processed with Can-Eye to estimate the $\mathrm{PAI}_{\text {eff }}$ for each crop.

\subsubsection{Image processing}

Conversely to methods designed to process upward-looking image using a threshold value for brightness in a single band (generally blue band) to identify gaps in the canopy (Leblanc et al., 2005), Can-Eye freeware (available at WWW1 ${ }^{1}$ ) used in this work is based on a RGB color classification of the image to discriminate vegetation elements from background (i.e., gaps). This approach allows exploiting downward-looking photographs for short canopies (background=soil) as well as upward-looking photographs for tall canopies (background = sky).

Can-Eye software processes simultaneously the set of $N=13$ images acquired over the same ESU. Note that the $N$ images were acquired with similar illumination conditions to limit the variation of color dynamics between images. A set of 13 photographs was processed within $30 \mathrm{~min}$ with a $2.5 \mathrm{GHz}$ PC with 2 Go of RAM. The processing is achieved in 3 main steps. First, image preprocessing is performed, which includes removing undesired objects (e.g. operator, sun glint) and

${ }^{1}$ Can Eye software, www.avignon.inra.fr/can_eye. 
image contrast adjustments to ensure a better visual discrimination between vegetation elements and background. Second, an automatic classification ( $k$-means clustering) is applied to reduce the total number of distinctive colors of the image to 324 which is sufficient to ensure accurate discrimination capacities while keeping a small enough number of colors to be easily manipulated. Finally, a default classification based on predefined color segmentation is first proposed and then iteratively and interactively refined by the user. The allocation of the colors to each class (vegetation elements versus background) is the most critical phase that needs to be interactive because colors depend both on illumination conditions and on canopy elements. At the end of this process a binary image, background versus vegetation elements (including both green and non-green elements) is obtained.

Can-Eye manages mixed pixels as follows: unclassified pixels are assumed to be mixed with fractions of each class depending on the location of the pixel in the color space. Note, that the frequency of mixed pixels is reduced with current high resolution digital camera particularly over most crops under study, which contain relatively large vegetation elements (Frazer et al., 2001; Jonckheere et al., 2004; Leblanc et al., 2005). It may, however, increase for crops composed of rather small gaps.

The RGB classification process potentially allows discriminating between green and non-green (e.g. senescent leaves) elements in the canopy in order to derive green LAI. In this case, three classes are used: green vegetation, non-green elements and background. Non-green elements and background classes are then merged together and considered as gaps in the canopy to derive only green LAI. While this feature represents a potential advantage over LAI-2000 and AccuPAR measurements, it requires diffuse illuminations to limit classification errors. However, it is still too uncertain to be used in this work because non-green elements tend to cover green vegetation, leading to underestimation of green LAI.

\subsubsection{Inversion of estimated gap fraction}

2.3.3.1. Effective PAI. To derive the spatial distribution of gap fraction, the $N$ binary hemispherical images are divided into $N_{\theta}=24$ concentric rings between $0^{\circ}$ and $60^{\circ}$ zenith angle with a zenith resolution of $\Delta \theta=2.5^{\circ}$, which are further subdivided into $N_{\varphi}=72$ cells of $\Delta \varphi=5^{\circ}$ in azimuth angle. The $60^{\circ}$ limit was selected to avoid using image edges containing increasing number of mixed and masked pixels. Further, these very oblique directions are more rapidly 'saturated' than the more vertical directions, leading to larger uncertainties at large PAI values. The gap fraction of each cell is computed as the ratio of the number of background pixels over the total number of pixels of the cell. Then, gap fraction is derived for each zenithal ring by averaging the cell gap fractions over the $\mathrm{N}$ classified images and the $N_{\varphi}$ azimuth cells of each ring, considering only the non-masked pixel. In the averaging process, the gap fraction of each cell is weighted by the proportion of non-masked pixel within the cell. The reason for averaging over the $\mathrm{N}$ images of the ESU (baseline retrieval) is to get a gap fraction measurement representative of the canopy heterogeneity (Weiss et al., 2004, cf. Section 3) and to avoid local artifacts such as a big leave masking the whole field-ofview of the camera and resulting in undefined logarithm of zero gap fraction. Can-Eye also provides gap fraction measurements and the estimated LAI (from Wilson method only) for each photograph. However, this information will be only used here to evaluate the sensitivity of DHP PAI eff $_{\text {estimates at }}$ the ESU scale to scaling methods (cf. Sections 3 and 4.1.3.4). In the rest of the manuscript, baseline retrievals will be used for the reasons given above. $\mathrm{PAI}_{\text {eff }}$ estimation by Can-Eye can be performed according to two modes.

In the first mode, the gap fraction measurements in each zenithal ring of the images are used to derive PAI $\mathrm{Pff}_{\text {and }}$ ALIA $\mathrm{eff}_{\mathrm{f}}$ from Eq. (1), assuming an ellipsoidal LIDF, and using the same LUT inversion technique (Weiss et al., 2004) described for LAI2000 in Section 2.1. The LUT relates all the combinations of $\mathrm{PAI}_{\text {eff }}(0-10$, step 0.01$)$ and ALIA $_{\text {eff }}\left(10-80^{\circ}\right.$, step $\left.2^{\circ}\right)$ to the gap fraction values simulated in each image zenithal ring. The $\mathrm{PAI}_{\text {eff }}$ and $\mathrm{ALIA}_{\text {eff }}$ solutions are chosen as the LUT element minimizing the following cost function:

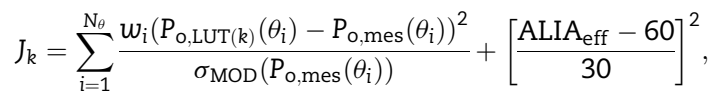

In Eq. (8), $P_{o, L U T(k)}$ and $P_{o, m e s}\left(\theta_{i}\right)$ are the simulated and measured, respectively, gap fraction in the direction $\theta_{i}$. The weight $w_{i}$ is the fraction of non-masked pixel within each ring over the $N$ images. $\sigma_{\mathrm{MOD}}\left(P_{\mathrm{o}, \mathrm{mes}}\left(\theta_{\mathrm{i}}\right)\right)$ is the "modeled" standard deviation of $P_{\varrho}$,mes $\left(\theta_{i}\right)$ computed over the $N$ images for each zenithal ring $i$; it is obtained by fitting a second order polynomial on its empirical value $\sigma\left(\mathrm{P}_{\mathrm{o} \text {,mes }}\left(\theta_{i}\right)\right)$ to smooth out the zenithal variation of $\sigma\left(\mathrm{P}_{\mathrm{o}, \mathrm{mes}}\left(\theta_{\mathrm{i}}\right)\right)$. The second term of Eq. (8) is a regularization term (Combal et al., 2002), that imposes constraints on the retrieved $A_{L A} A_{\text {eff }}$ values (assuming that $\operatorname{ALA}_{\text {eff }}=60^{\circ} \pm 30^{\circ}$ ).

In the second mode, single direction gap fraction at $\theta=57.5^{\circ}$, computed here for $56^{\circ}$ to $59^{\circ}$ zenith angle ring, is used to retrieve $\mathrm{PAI}_{\text {eff }}$ according to Eq. (3) (Wilson method).

2.3.3.2. Clumping index and PAI estimation. Key advantage of DHP compared to LAI-2000 and AccuPAR instruments is the ability to account for vegetation clumping by modifying Eq. (1) as follows (Nilson, 1971),

$P(\theta)=\left[\exp \frac{-\Omega(\theta) \cdot G(\theta) \cdot P A I}{\cos (\theta)}\right]$,

where $\Omega(\theta)$ is the clumping index in the direction $\theta$. Eq. (9) is called modified Poisson model. The logarithm gap fraction averaging approach (Lang and Xiang, 1986) is implemented in Can-Eye to compute $\Omega(\theta)$. In this method, canopy elements are assumed to be randomly distributed within each individual cell. The size of individual cells should be large enough so that the statistics of the gap fraction are meaningful and small enough so that the assumption of randomness of leaf distribution within the cell is valid. In this work, we selected $\Delta \theta=2.5^{\circ}$ and $\Delta \varphi=5^{\circ}$ as advised by the authors of Can-Eye $\left(W W W 1^{1}\right)$. The gap fraction, as well as its logarithm, are computed for each cell and then averaged over azimuth $\varphi$ and over the $\mathrm{N}$ images for each zenithal ring. Note that averaging over $\mathrm{N}$ images properly distributed to sample canopy variability is critical to quantify clumping at the canopy-scale (e.g. row effect). The averaging process is weighted by the frequency of non-masked pixel in each cell. If there is no gap in the cell (only vegetation, i.e., $P=0$ ), $P$ is assumed to 
be equal to $P_{o, \text { sat }}$ (saturation) derived from Eq. (1) using a prescribed $\mathrm{LAI}_{\text {sat }}$ value (here $\mathrm{LAI}_{\text {sat }}=10$ ) and $\mathrm{ALIA}_{\text {eff }}$ as previously estimated from the LUT. The ratio between the average of the logarithm of the gap fraction and the logarithm of the average of the gap fraction provides an estimation of the clumping index for each zenithal ring:

$\Omega\left(\theta_{i}\right)=\frac{(1 / N) \sum_{j=1}^{N}\left(1 / N_{\varphi}\right) \sum_{\varphi=1}^{N_{\varphi}} \log \left(P_{\varphi}\left(\theta_{i}\right)\right)}{\log \left[(1 / N) \sum_{j=1}^{N}\left(1 / N_{\varphi}\right) \sum_{\varphi=1}^{N_{\varphi}}\left(P_{\varphi}\left(\theta_{i}\right)\right)\right]}$.

After computing $\Omega\left(\theta_{\mathrm{i}}\right)$, PAI and ALIA are estimated from Eq. (9) using the same LUT inversion technique described above for $\mathrm{PAI}_{\text {eff }}$.

\section{Experimental site and data sampling}

The experiment was performed during March 3-5, 2005 at the Instituto Nacional Tecnologia Agropecuaria (INTA) Estación Experimental Agropecuaria (EEA) Manfredi site in Cordoba province, Argentina ( $\left.31^{\circ} 52^{\prime} 3^{\prime \prime} \mathrm{S}, 63^{\circ} 44^{\prime} 20^{\prime \prime} \mathrm{W}\right)$. The field work was done in conjunction with the Committee on Earth Observing Satellites (CEOS) Working Group on Calibration and Validation (WGCV) plenary meeting in Argentina $\left(W W W 2^{2}\right)$. The site covers an area of approximately $2.5 \mathrm{~km} \times 5 \mathrm{~km}$ and is made of various agricultural fields with $0.5 \mathrm{~km} \times 0.5 \mathrm{~km}$ typical size (Fig. 1). The main crop types were soybean, corn, alfalfa, sorghum, peanut and pasture. Overall, the site exhibits substantial spatial heterogeneity due to species composition and varying stages of growth and senescence, resulting in substantial within- and betweenspecies LAI variations.

Data sampling in this study was designed according to CEOS standard procedure (Morisette et al., 2006) for the validation of moderate resolution $(\sim 1 \mathrm{~km})$ satellite biophysical products. This procedure consists in establishing a relationship between high spatial resolution satellite data (here $30 \mathrm{~m}$ pixel) and ground LAI measurement to scale-up local LAI in situ measurements, and produce a high spatial resolution LAI map over the whole site. This map is ultimately aggregated to be compared with coarse resolution satellite data. Thus, LAI ground measurements must be representative of a high spatial resolution pixel, called hereafter ESU. Measurement sampling scheme within each ESU was implemented in the form of a cross, consisting of 13 points, $5 \mathrm{~m}$ apart. To properly sample row effects and the variability along rows in discontinuous canopies, the cross was oriented at $45^{\circ}$ from the row direction with its center located at the middle of the row. This sampling scheme has been proven to be efficient to capture the canopy spatial heterogeneity (Tan et al., 2005; Morisette et al., 2006; Baret et al., submitted for publication). Besides, intercomparison of $\mathrm{PAI}_{\text {eff }}$ retrieval methods at the ESU scale is more consistent than at the point scale, since errors due to differences in instrument footprint and temporal registration of point location are substantially reduced at the ESU scale (Fernandes et al., 2003). Note also that at the ESU

\footnotetext{
2 23-d WGCV plenary meeting in Argentina, http://wgcv.ceos.org/documentation/wgcv23.htm.
}

scale, the random errors associated with each retrieval technique should cancel out, leaving only the systematic bias associated with $\mathrm{PAI}_{\text {eff }}$ estimation.

Two methods can be used to scale $\mathrm{PAI}_{\mathrm{eff}}$ from point to ESU. In Method 1, $\mathrm{PAI}_{\text {eff }}$ is computed from transmittance measurements at each point of the ESU and then point estimates are averaged over the ESU. In Method 2, transmittance is first averaged over the ESU and then $\mathrm{PAI}_{\text {eff }}$ is estimated from the mean ESU transmittance. Because of the non-linear relationship between transmittance and $\mathrm{PAI}_{\text {eff }}$ (cf. Eqs. (1) and (4)), results from Methods 1 and 2 will be different over heterogeneous canopies (Lang and Xiang, 1986). As demonstrated by Garrigues et al. (2006), $\mathrm{PAI}_{\text {eff }}$ scaling bias between both methods is proportional to the spatial variability of gap fraction (caused by canopy spatial heterogeneity and measurement errors) and the degree of non-linearity of the PAI $\mathrm{Pff}_{\mathrm{ff}}$ retrieval model. The degree of non-linearity is quantified by the second derivative of the retrieval model which is a decreasing function of transmittance for the models investigated in this paper. Differences between Methods 1 and 2 will be evaluated for each instrument in relation with canopy spatial heterogeneity (cf. Section 4). However, the proper scaling method is still under debate. As advised by some authors (LI-COR, 1991; Hyer and Goetz, 2004), Method 1 is the mathematically correct way to scale-up PAI $_{\text {eff }}$ from point to stand because it averages the logarithm of transmission which is linearly related to $\mathrm{PAI}_{\text {eff. }}$. Besides, since the random distribution of canopy elements is generally more valid over small area (point scale) that at larger scale (ESU scale), $\mathrm{PAI}_{\text {eff }}$ estimated from Method 1 should be less affected by vegetation

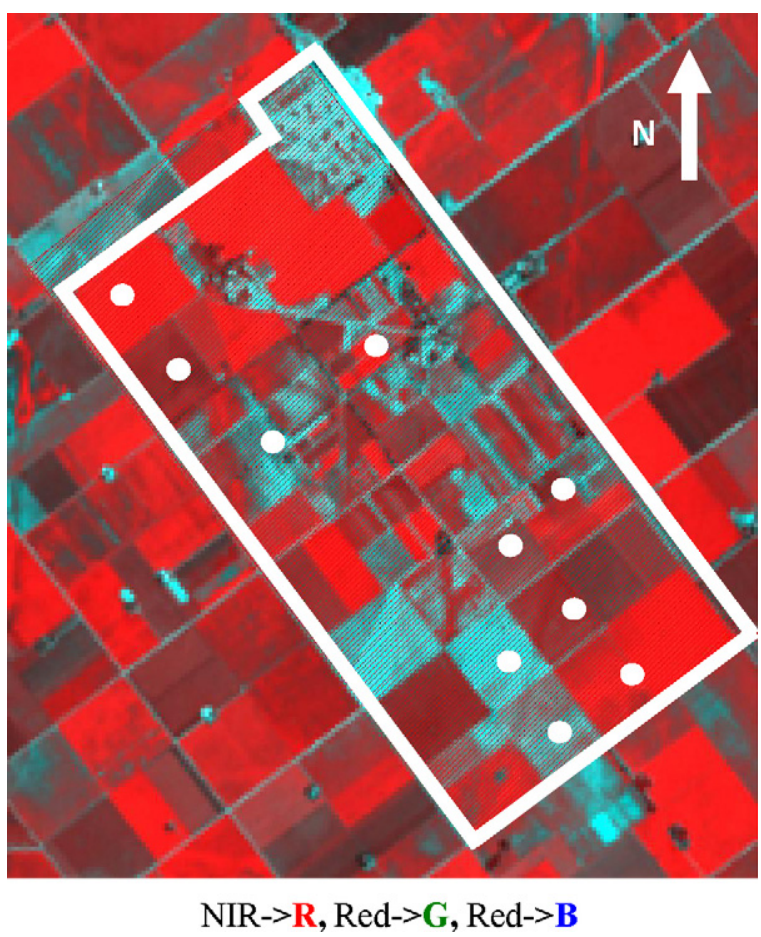

Fig. 1 - Location of the Manfredi site (white border) and the Elementary Sampling Units (ESU, white dots) on the falsecolor image derived from near-infrared (NIR) and red spectral bands of ALI sensor onboard of EO-1 platform (21 January 2005, WRS2 229/082, 30-m). 


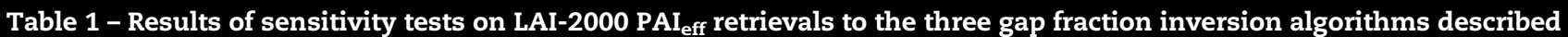
in Section 2.1: the standard method (Stand.) implemented in LAI-2000 software, the Wilson (Wils.) method and the LUT method

\begin{tabular}{|c|c|c|c|c|c|c|c|c|}
\hline \multirow[t]{2}{*}{ Methods } & \multicolumn{4}{|c|}{ Diffuse illumination } & \multicolumn{4}{|c|}{ Direct illumination } \\
\hline & $R^{2}$ & RMSE & BIAS & SDD & $R^{2}$ & RMSE & BIAS & SDD \\
\hline Stand. vs. Wils. & 0.96 & $0.50(27)$ & $-0.33(-12)$ & $0.38(24)$ & 0.93 & $0.65(23)$ & $-0.19(-3)$ & $0.62(22)$ \\
\hline Stand. vs. LUT & 0.76 & $1.30(32)$ & $-0.49(-13)$ & $1.20(29)$ & 0.87 & $0.73(20)$ & $-0.03(-2)$ & $0.73(20)$ \\
\hline Wils. vs. LUT & 0.76 & $1.14(35)$ & $-0.16(-6)$ & $1.13(34)$ & 0.89 & $0.77(40)$ & $0.16(-5)$ & $0.76(40)$ \\
\hline
\end{tabular}

The analysis is conducted at the point scale (33 samples). For comparison of algorithm $x$ versus $y$, BIAS represents the mean difference between $x$ and $y$ retrievals and SDD is the standard deviation of the difference between $x$ and $y$ retrievals (quantifying the random fluctuations between $\mathrm{x}$ and $\mathrm{y}$ retrievals). Note that BIAS and SDD are two components of the RMSE linked by $\mathrm{RMSE}^{2}=\mathrm{SDD}^{2}+\mathrm{BIAS}^{2}$. These metrics are also computed relatively to $\mathrm{x}$ retrievals and shown in round parenthesis (\% values) in each cell.

clumping. However, as demonstrated by Lang and Xiang (1986), the determination of $\mathrm{PAI}_{\text {eff }}$ from the logarithm of the transmission measured at one point may be uncertain due to possible invalidity of light transmission model assumptions, lack of spatial representativeness of canopy structure and possible undefined logarithm of zero transmittance. Besides, Weiss et al. (2004) demonstrated that Method 2 provides the most representative effective $\mathrm{PAI}_{\text {eff }}$ estimate at the ESU scale. For this reason and for consistency with DHP baseline retrievals based on Method 2, Method 2 will be used here for analyzing $\mathrm{PAI}_{\text {eff }}$ sensitivity to illumination conditions (Section 4.1) as well as for intercomparison of $\mathrm{PAI}_{\text {eff }}$ estimates from each instrument (Section 4.2).

Ten ESUs were established across the site (Fig. 1) to sample LAI variability over each crop type: one point for alfalfa, two for corn, three for soybean, two for sorghum, one for peanut and one for pasture. Note that only DHP measurements were performed over peanut and pasture because LAI-2000 and AccuPAR acquisitions were unfeasible due to too short canopies. All ESUs were located in the middle of the field with special attention paid to avoid roads or other non-canopy objects falling in the sensor's field of view. LAI-2000, AccuPAR and DHP measurements were performed simultaneously, under diffuse illumination (about sunrise or sunset) and direct sunlight illumination (morning and/or afternoon measurements).

\section{Results}

The sensitivity analysis to retrieval methods for LAI-2000 and parameters for AccuPR was performed at the point measurement scale to benefit from a larger number of samples. This was not possible for DHP because Can-Eye only provides estimates from the LUT method at the ESU scale. For the sensitivity analysis to illumination conditions and spatial heterogeneity as well as cross-sensor $\mathrm{PAI}_{\text {eff }}$ intercomparison, retrievals at the ESU scale were used for the reasons given above.

\subsection{Sensitivity analysis of individual sensors}

\subsubsection{LAI-2000}

4.1.1.1. Sensitivity to gap fraction inversion methods. LAI-2000 $\mathrm{PAI}_{\text {eff }}$ retrievals from standard, Wilson, and LUT methods (cf. Section 2.1) were compared at the point scale (Table 1 and
Fig. 2). Results show that retrievals from the standard method are generally lower than those from the LUT and Wilson methods (note the negative bias reported in Table 1), especially at high $\mathrm{PAI}_{\text {eff }}$ and under diffuse conditions (Fig. 2). Indeed, transmittance in ring 5 can be substantially increased by multiple scattering of light within the canopy as compared to other rings, decreasing thus the estimation of $\mathrm{PAI}_{\text {eff }}$ (Leblanc and Chen, 2001). This effect is amplified by the large weight given to the fifth ring transmittance in the standard retrieval method (cf. Section 2.1), explaining the negative bias between $\mathrm{PAI}_{\text {eff }}$ estimates from the Standard method and those from the LUT. Note that the negative bias of $12 \%$ between standard and Wilson retrievals under diffuse condition is similar to that reported by Chen et al. (1997) and Leblanc and Chen (2001) over forests. The largest discrepancy (cf. RMSE in Table 1) is observed between the LUT retrievals and those from other algorithms. LUT estimates are particularly larger than others at high $\mathrm{PAI}_{\text {eff }}$ and under diffuse conditions (cf. Fig. 2). This may indicate that the LUT algorithm is less affected by transmit-

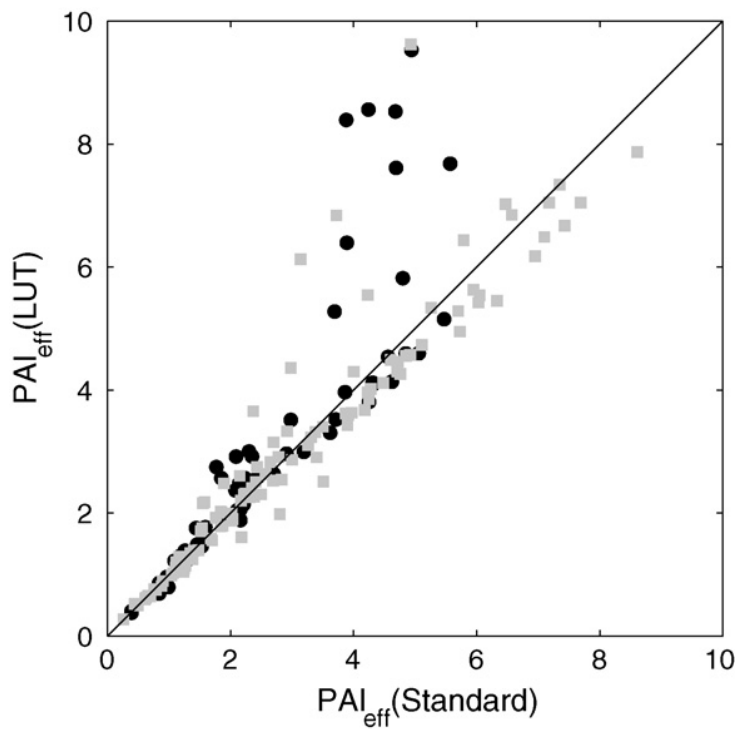

Fig. 2 - Comparison between LAI-20000 $\mathrm{PAI}_{\text {eff }}$ estimated from the standard method implemented in LAI-2000 software and the LUT method proposed by Weiss et al. (2004). The analysis is conducted at the point scale and corresponding RMSE, $r^{2}$ values are reported in Table 1. Measurements under direct and diffuse illumination are represented by grey squares and black circle, respectively. 
tance saturation as compared to the standard method. LAI2000 retrievals from the LUT method will thus be selected for the cross-instrument $\mathrm{PAI}_{\text {eff }}$ intercomparison in Section 4.2.

The same analysis conducted at the ESU scale (not presented here for sake of concision) shows that while the bias between methods remains, most random fluctuations between retrievals cancel out $\left(r^{2}>0.9\right.$ and on average RMSE at the ESU scale are reduced by $22 \%$ and $57 \%$ under diffuse and direct illuminations, respectively). Note, however, that the absolute accuracy of these methods is not considered here, emphasis being put on their consistency and departure.

4.1.1.2. Sensitivity to illumination conditions. The magnitude of sensitivity to illumination conditions at the ESU scale (relative RMSE 26-29\%, cf. Fig. 3a) is similar to that reported by other studies (for example, $20 \%$ over forest; Leblanc and Chen, 2001). Literature (LI-COR, 1991; Leblanc and Chen, 2001) indicates that LAI-2000 retrievals are, in general, inaccurate under direct sunlight because of high contamination of measurements by leaf specular reflection, causing underestimation of $\mathrm{PAI}_{\text {eff }}$ (mainly at large $\mathrm{PAI}_{\text {eff }}$, cf. Fig. 3a). Besides, in direct sunlight conditions, the ring containing the sun actually measures the gap fraction mainly for the sun zenithal and azimuthal particular direction, resulting in a very poor spatial representativeness and providing unreliable $\mathrm{PAI}_{\mathrm{eff}}$ estimation (Hyer and Goetz, 2004). Note, also, that under direct sunlight LAI-2000 measurements are highly sensitive to slight deviation of sensor from horizontal positioning. While Leblanc and Chen (2001) proposed a method to correct direct sunlight effects, its application was not feasible in this work because the range of solar zenith angles was limited.

Differences between diffuse and direct illuminations are larger for short canopies with smaller leaves (e.g. soybean) than for taller canopy with larger leaves (e.g. corn) for which most of the sunlit foliage is hidden from the sensor's view.

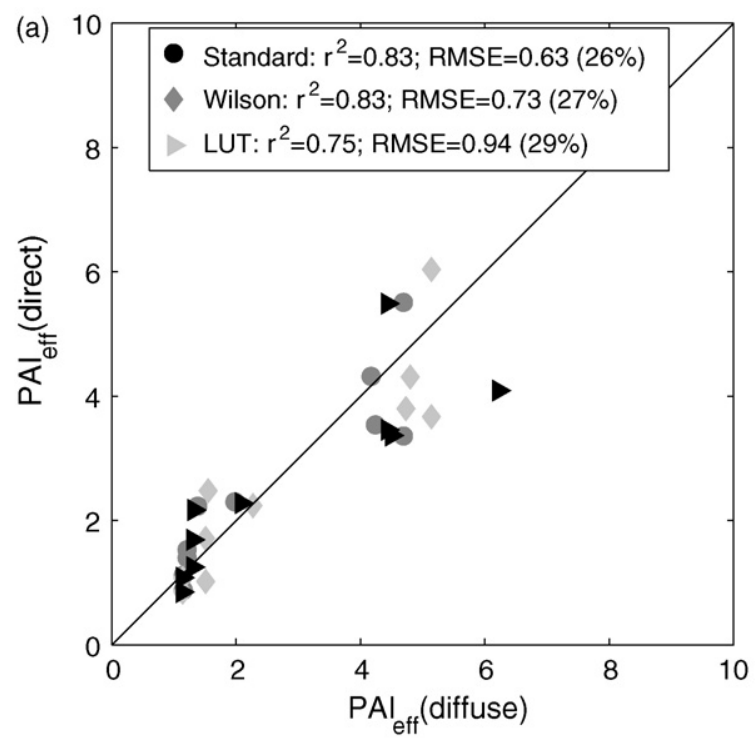

Retrievals from the LUT method show the largest sensitivity to illumination conditions. This is mainly due to rings 1 and 2, which are more sensitive to direct sunlight radiation than other rings because of the larger gap fraction observed at these low zenith angles, and the larger number of sunlit leaves directly viewed by the sensor at these rings. Smaller sensitivity is observed for estimates from the Wilson method. Indeed, this method relies on measurements in ring 4 from which less sunlit leaves are viewed by the sensor since they tend to be masked by lower foliage layers. However, retrievals from the standard method show smaller RMSE than that of LUT retrievals while both methods capitalize on measurements from the five rings. Indeed, the standard method places a heavy emphasis on the input from ring 5 which have the least influence from direct sunlight because the sun is generally higher than $61^{\circ}$ from zenith in our measurements.

4.1.1.3. Sensitivity to scaling methods. Fig. $3 \mathrm{~b}$ compares $\mathrm{PAI}_{\mathrm{eff}}$ estimates at the ESU scale using scaling Method 1 to those derived from Method 2. As expected, Method 1 provides larger estimates than Method 2 because $\mathrm{PAI}_{\text {eff }}$ is a convex and nonlinear function (inverse of Eq. (1)) of gap fraction (Garrigues et al., 2006). Differences in $\mathrm{PAI}_{\text {eff }}$ between Methods 1 and 2 reflect variability of gap fraction measurements over the ESU due to canopy heterogeneity (especially for discontinuous canopies) and possible measurement errors. Note, however, that these differences are relatively small because of the low spatial heterogeneity of the crop canopies investigated here. Scattering between Methods 1 and 2 slightly increases at large $\mathrm{PAI}_{\text {eff, }}$, corresponding to short canopies (soybean crop) for which LAI-2000 measurements are unstable (cf. Section 4.2) and thus very variable over the ESU. Besides, the very low transmittance measured over soybean crops increases the degree of non-linearity of the algorithm (cf. Section 3) that amplifies the $\mathrm{PAI}_{\text {eff }}$ scaling bias. The higher RMSE observed for

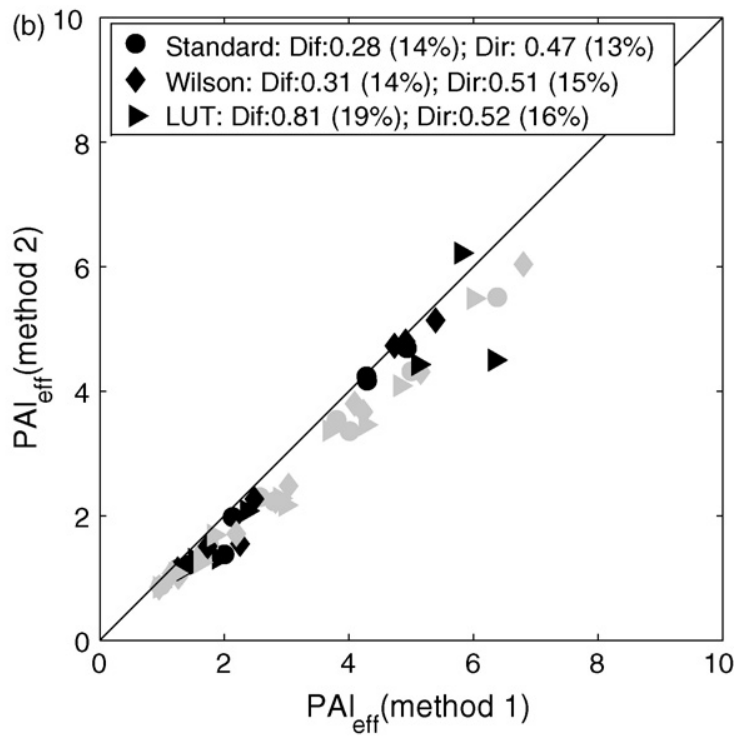

Fig. 3 - Sensitivity of LAI-2000 $\mathrm{PAI}_{\text {eff }}$ retrievals to (a) illumination conditions (diffuse versus direct sunlight) and (b) scaling method (Method 1 versus Method 2, as described in Section 3). Values in round parenthesis indicate RMSE relatively to the variable shown in the $x$-axis. The analysis is conducted at the ESU scale. In sub-part (b) (only), retrievals under diffuse (direct) illumination are shown in black (grey) and corresponding RMSE is shown first (second) in the caption windows. 
the LUT method may be due to a higher degree of non-linearity associated with this inversion technique as compared to the other retrieval methods. Scattering between Methods 1 and 2 is, on average, larger under direct illuminations than diffuse illumination. This is caused by measurement artifacts generated by direct sun beam, increasing the variability of gap fraction over the ESU.

\subsubsection{AccuPAR}

4.1.2.1. Sensitivity analysis to retrieval parameters. In contrast to LAI-2000 retrieval technique, AccuPAR algorithm requires a larger number of parameters either measured by the sensor $\left(f_{b}\right)$ or provided by the user (canopies parameters $x$ and $a$ ). These parameters are potential sources of uncertainties in $\mathrm{PAI}_{\text {eff }}$ estimation. We follow the protocol developed by Hyer and Goetz (2004) for conifer forest to test the sensitivity of $\mathrm{PAI}_{\text {eff }}$ to these parameters over several crop types. Emphasis will be put on the dependence of parameter sensitivity to illumination conditions (direct versus diffuse).

Sensitivity to fraction of direct beam $f_{b}$. Uncertainty in estimation of the ratio of direct to total flux, $f_{b}$, may result from measurement errors or rapid changes in illumination conditions during data acquisition (Hyer and Goetz, 2004). To assess the sensitivity of $\mathrm{PAI}_{\text {eff }}$ to $f_{\mathrm{b}}$, we artificially introduced an error $\delta e$ to the actual $f_{\mathrm{b}}$ value measured by the ceptometer for each point. We tested $\delta e= \pm 0.1$ and \pm 0.2 which correspond to possible levels of error as reported by Hyer and Goetz (2004). We distinguish between diffuse (small $f_{\mathrm{b}}$ value, here $f_{\mathrm{b}}<0.3$ ) and direct (corresponding to large $f_{b}$ value, here $f_{b}>0.7$ ) illumination conditions. Note, that when $f_{\mathrm{b}}+\delta e$ is larger than 1 or smaller than 0 the point is not selected in the analysis. Fig. 4 and Table 2 indicate that sensitivity of $\mathrm{PAI}_{\text {eff }}$ retrieval to $\mathrm{f}_{\mathrm{b}}$ generally increases with the magnitude of $\delta e$, and is larger under direct illumination (relative RMSE 8-24\%) than under diffuse illumination (relative RMSE 4-8\%). These findings confirm similar ones obtained by Hyer and Goetz (2004) over coniferous forest. While this result was predicted by theory (Eq. (4) and (5)), it show that the sensitivity of $\mathrm{PAI}_{\text {eff }}$ retrievals to

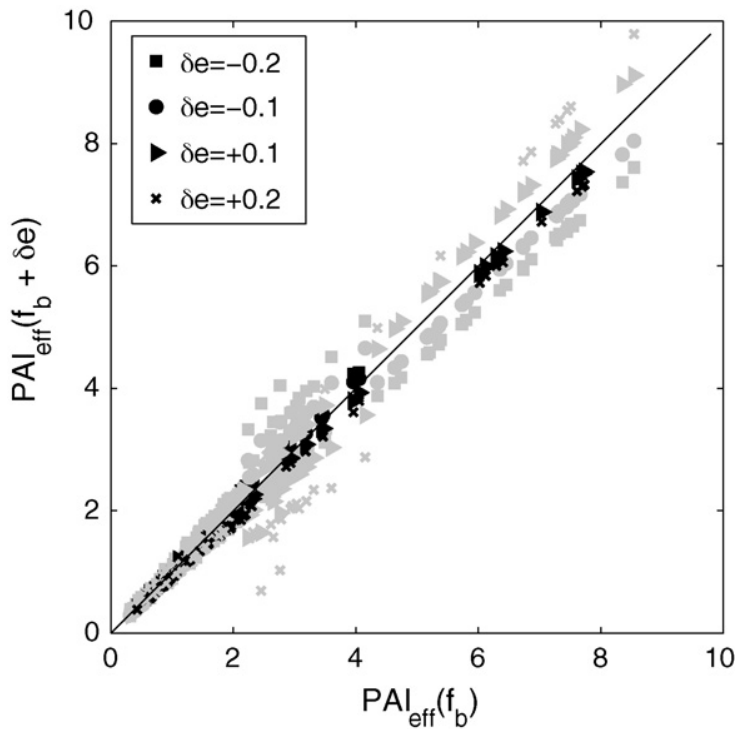

Fig. 4 - Sensitivity analysis of AccuPAR PAI ${ }_{\text {eff }}$ retrievals to error $\delta e$ in $f_{b}$ (ratio of direct to total flux parameter) measured by AccuPAR. The analysis is conducted at the point scale. Retrievals under diffuse (direct) illumination are shown in black (grey) and corresponding RMSE are reported in Table 2 .

$f_{\mathrm{b}}$ error is still low enough to be neglected under non-perfect diffuse conditions $\left(f_{\mathrm{b}} \neq 0\right)$. Besides, Fig. 4 additionally indicates that the discrepancies in $\mathrm{PAI}_{\mathrm{eff}}$ introduced by $f_{\mathrm{b}}$ measurement errors are not systematic under direct illumination (low bias and larger random fluctuation SDD, cf. Table 2). Indeed, the magnitude of the sensitivity is low at $\mathrm{PAI}_{\text {eff }}$ smaller than 2. For $\mathrm{PAI}_{\text {eff }}$ between 2 and 4, it slightly increases for some points corresponding to an overestimation (underestimation) of $\mathrm{PAI}_{\text {eff }}$ for negative (positive) errors $\delta$. For $\mathrm{PAI}_{\text {eff }}$ larger than 4 , the sensitivity is larger for all points but with an under-

Table 2 - Results of sensitivity tests on AccuPAR PAI ${ }_{\text {eff }}$ retrievals to key parameters: ratio of direct to total flux $f_{b}$; leaf angle parameter $\mathrm{x}$; and leaf absorptivity $a$

\begin{tabular}{|c|c|c|c|c|c|c|c|c|}
\hline \multirow[t]{2}{*}{ Parameters } & \multicolumn{4}{|c|}{ Diffuse illumination } & \multicolumn{4}{|c|}{ Direct illumination } \\
\hline & $R^{2}$ & RMSE & BIAS & SDD & $R^{2}$ & RMSE & BIAS & SDD \\
\hline \multicolumn{9}{|c|}{$f_{\mathrm{b}}$ (baseline) vs. $f_{\mathrm{b}}+\delta e$} \\
\hline$\delta e=-0.2$ & 1.00 & $0.13(7)$ & $-0.12(-7)$ & $0.06(1)$ & 0.96 & $0.51(16)$ & $0.07(-2)$ & $0.50(15)$ \\
\hline$\delta e=-0.1$ & 1.00 & $0.07(4)$ & $-0.06(-4)$ & $0.03(1)$ & 0.99 & 0.27 (8) & $0.04(-1)$ & $0.27(8)$ \\
\hline$\delta e=+0.1$ & 1.00 & $0.09(4)$ & $0.08(3)$ & $0.05(2)$ & 0.99 & $0.32(10)$ & $-0.05(2)$ & $0.31(10)$ \\
\hline$\delta e=+0.2$ & 1.00 & $0.19(8)$ & $0.16(8)$ & $0.11(4)$ & 0.93 & $0.78(24)$ & $0.05(7)$ & $0.78(22)$ \\
\hline \multicolumn{9}{|c|}{$x=1$ (baseline) vs. $x=x_{\text {new }}$} \\
\hline$x_{\text {new }}=0.5$ & 1.00 & $0.03(2)$ & $0.02(1)$ & $0.02(1)$ & 0.99 & $1.00(21)$ & $-0.56(-12)$ & $0.83(17)$ \\
\hline$x_{\text {new }}=1.5$ & 1.00 & $0.03(2)$ & $-0.03(-2)$ & $0.02(1)$ & 0.99 & $0.47(10)$ & $0.23(4)$ & $0.41(9)$ \\
\hline \multicolumn{9}{|c|}{$a=0.9$ (baseline) vs. $a=a_{\text {new }}$} \\
\hline$a_{\text {new }}=0.64$ & 1.00 & $0.62(20)$ & $-0.47(-20)$ & $0.39(1)$ & 1.00 & $0.71(20)$ & $-0.57(-20)$ & $0.42(0)$ \\
\hline$a_{\text {new }}=1$ & 1.00 & $0.17(5)$ & $0.13(5)$ & $0.11(1)$ & 1.00 & $0.19(5)$ & $0.16(5)$ & $0.12(0)$ \\
\hline
\end{tabular}

The analysis is done compared to baseline retrievals and is conducted at the point scale ( 33 samples). BIAS represents the mean difference between baseline retrievals and modified algorithm retrievals and SDD is the standard deviation of the difference between baseline and modified algorithm retrievals. These metrics are also computed relatively to baseline value shown in round parenthesis (\% values) in each cell. 
estimation (overestimation) of $\mathrm{PAI}_{\mathrm{eff}}$ for negative (positive) errors $\delta$.

Sensitivity to leaf angle distribution parameter $x$. The ceptometer uses the default value of $x=1$ corresponding to a spherical leaf angle distribution. It is a reasonable approximation for most canopies under study including alfalfa and sorghum but the leaf angle distribution of corn and soybean is frequently erectophile and planophylle, respectively. We will thus evaluate here the sensitivity of $\mathrm{PAI}_{\text {eff }}$ estimation to two distinct types of foliage, namely erectophile $(x=0.5)$ and planophile $(x=1.5)$ foliage. Table 2 shows that the sensitivity is much lower under diffuse conditions (relative RMSE 2\%) than under direct illumination (relative RMSE 10-21\%), in agreement with the theory (Eq. (5)) even for non-perfect diffuse conditions. The standard value $(x=1)$ overestimate (by $4 \%$ ) and underestimate (by 12\%) $\mathrm{PAI}_{\text {eff }}$ values for planophile foliage $(x=1.5)$ and erectophile foliage $(x=0.5)$, respectively. This confirms results from Hyer and Goetz (2004) for coniferous forest. Since these differences are systematic, they will impact retrievals at the ESU scale.

Sensitivity to leaf absorptivity a. In AccuPAR retrieval algorithm, leaf optical properties are described using parameter $a$ which is a crude approximation of the underlying complex radiative processes at PAR wavelengths. To evaluate the impact of possible uncertainty associated with selection of parameter $a$ on $\mathrm{PAI}_{\text {eff }}$ retrievals, we compare baseline retrievals based on the default value $(a=0.9)$ with those, derived using $a=1$ (black foliage assumption) and $a=0.64$ used in Welles's (1990) retrieval model. Results (Table 2) indicate a positive bias of $5 \%$ (a negative bias of $20 \%$ ) between baseline retrievals and those derived using $a=1 \quad(a=0.64)$ under both diffuse and direct illuminations. These results are similar to those obtained by Hyer and Goetz (2004) for coniferous forest. They also show that conversely to $f_{\mathrm{b}}$ and $x$ parameters, the sensitivity to the parameter $a$ is similar under both diffuse and direct illuminations as theoretically expected since this parameter is involved in both Eq. (4) and (5). These biases will propagate at the ESU scale, affecting validation results.

While the baseline value of $a=0.9$ used in this work is a reasonable approximation for green leaves of most species under study (AccuPAR manual, Campbell and Norman, 1998), the 0.64 value is probably closer to optical properties of senescent leaves. Thus, in presence of senescent vegetation, as it is the case for corn and sorghum in this work, baseline AccuPAR retrievals may substantially underestimate $\mathrm{PAI}_{\text {eff. }}$.

4.1.2.2. Sensitivity to illumination conditions. Fig. 5a displays $\mathrm{PAI}_{\text {eff }}$ value under diffuse versus direct illumination conditions. Sensitivity to illumination conditions (relative RMSE $86 \%$ ) is higher than for LAI-2000 (relative RMSE 26-29\%) and increases with $\mathrm{PAI}_{\text {eff. }}$. As for LAI-2000, it is not systematic and is larger for short canopies with small leaves (e.g. soybean) than tall canopy with large leaves (e.g. corn). It is mainly the result of uncertainties in retrieval parameters $\left(f_{b}, x\right)$ that affect $P_{\text {Aff }}$ estimation under direct illumination and at high $\mathrm{PAI}_{\mathrm{eff}}$.

4.1.2.3. Sensitivity to scaling methods. Fig. 5b compares ESU $\mathrm{PAI}_{\text {eff }}$ estimates from scaling Methods 1 and 2. The magnitude of the differences between both methods (RMSE 4-9\%) is smaller for AccuPAR than for LAI-2000 (RMSE 13-19\%) while logarithm function of transmittance is involved in both retrieval techniques. This implies that transmittance measurements from LAI-2000 are more variable than those from AccuPAR over the ESU. Indeed, since AccuPAR integrates radiation along the sensor, it provides a better spatial sampling of gap fraction, and captures more canopy spatial heterogeneity at the point scale than LAI-2000. This characteristic is particularly important for crop with small gaps in the canopy. Note also that discrepancies between Methods 1
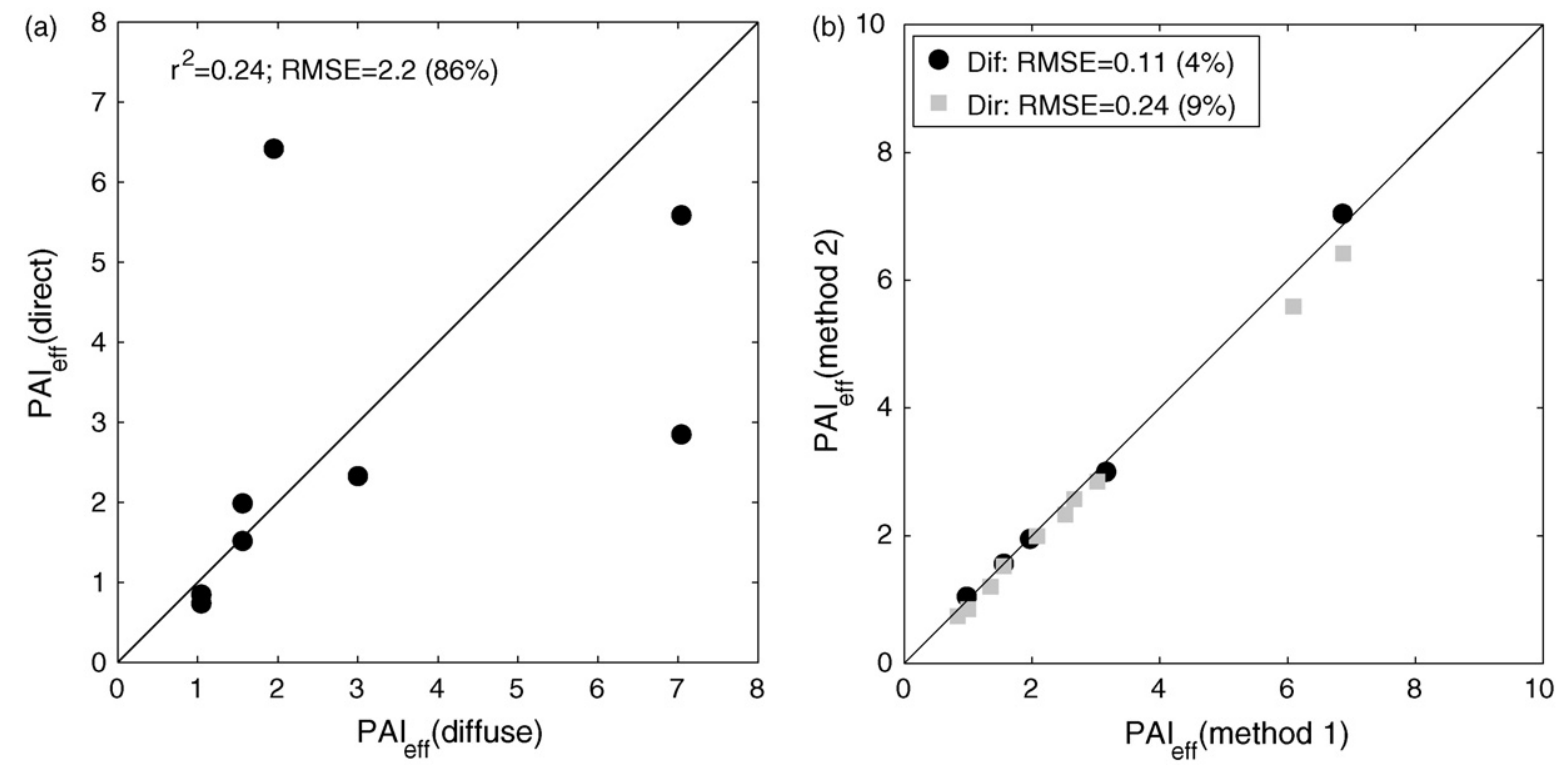

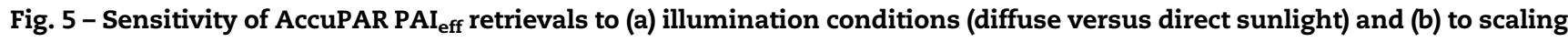
method (Method 1 versus Method 2). In Figure b, retrievals under diffuse and direct illuminations are shown in black circle and grey square, respectively. Values in round parenthesis indicate RMSE relatively to the variable shown on the $x$-axis. The analysis is conducted at the ESU scale. 

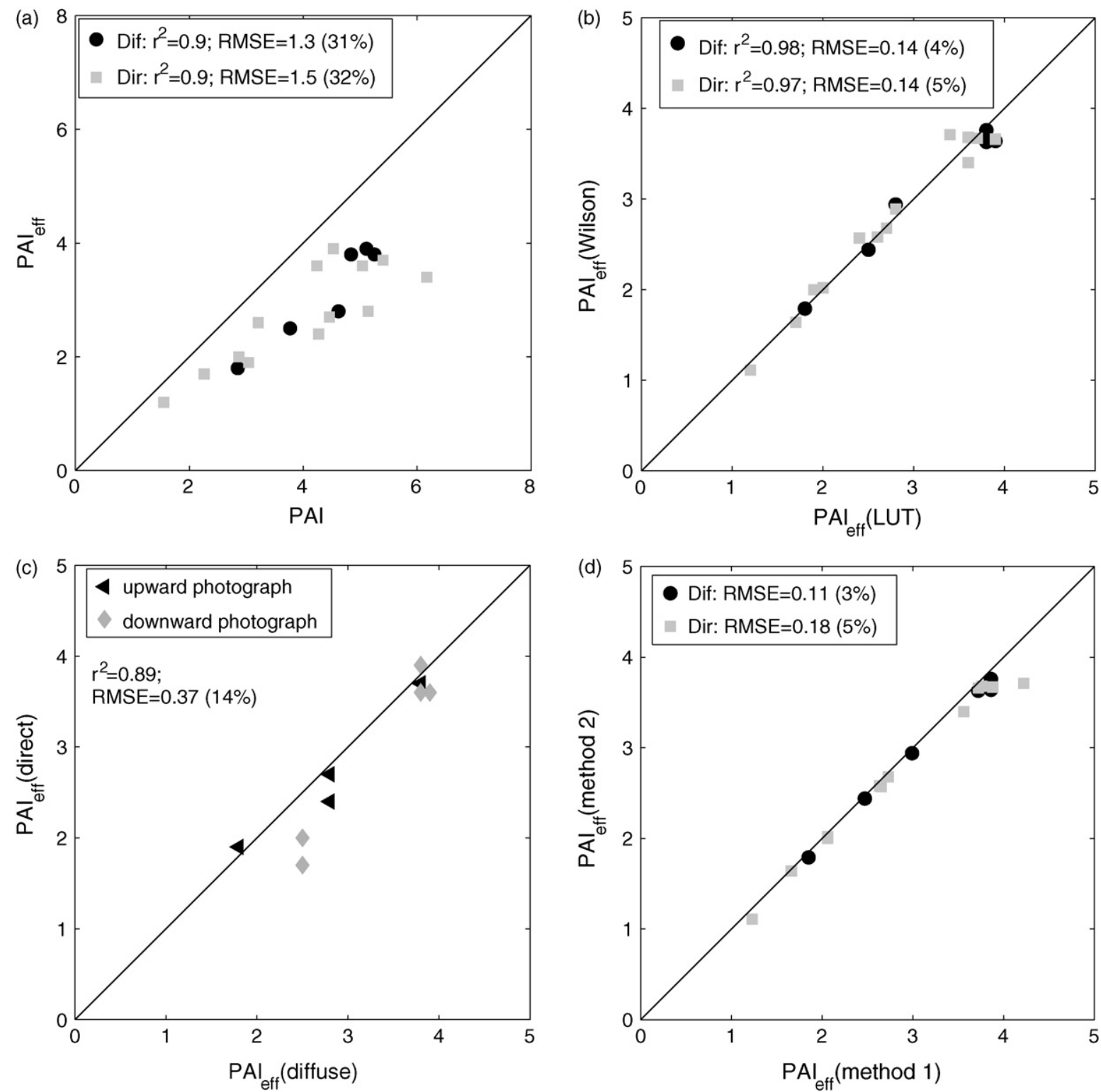

Fig. 6 - Sensitivity analysis of DHP retrievals conducted at the ESU scale. (a) Sensitivity to vegetation clumping, as captured by difference between $\mathrm{PAI}_{\text {eff }}$ and PAI. (b) Comparison of $\mathrm{PAI}_{\text {eff }}$ retrievals based on multiple directional gap fraction measurements (LUT method) and retrievals using gap fraction at single zenith angle $=57.5^{\circ}$ (Wilson method). (c) Comparison of $\mathrm{PAI}_{\text {eff }}$ retrievals under direct and diffuse illumination. (d) Sensitivity to scaling method (Method 1 versus Method 2) for Wilson method PAI $_{\text {eff }}$ retrievals. Values in round parenthesis indicate RMSE relatively to the variable shown on the $x$-axis. In sub-parts ( $a, b$, and d) (only), retrievals under diffuse and direct illuminations are shown in black circle and grey square, respectively.

and 2 are slightly larger under direct illuminations than diffuse illumination because of the larger variability of gap fraction point measurements under direct illumination.

\subsubsection{DHP}

4.1.3.1. Sensitivity to clumping. Fig. 6a compares PAI (corrected from foliage clumping) and $\mathrm{PAI}_{\text {eff }}$, both derived from DHP measurements. For all the canopies investigated, clumping index is less than one, and thus $\mathrm{PAI}_{\text {eff }}$ underestimates PAI. The clumping effect increases at $\mathrm{PAI}_{\text {eff }}$ larger than three corresponding to discontinuous canopies with row structure (corn). Note that the estimate of clumping magnitude is similar under diffuse and direct illuminations. The differences between $\mathrm{PAI}_{\text {eff }}$ and PAI (RMSE 31\%) are larger than those observed between scaling Methods 1 and 2 for LAI-2000 retrievals using the LUT inversion technique (RMSE 16-19\%). The Lang and Xiang (1986) method used to compute the clumping index in Can-Eye algorithm is equivalent to calculating the ratio between $\mathrm{PAI}_{\text {eff }}$ estimated from Method 1 (mean of the logarithm of gap fraction over the ESU) and 
$\mathrm{PAI}_{\text {eff }}$ estimated from Method 2 (logarithm of the mean gap fraction over the ESU). However, DHP imagery provides a far finer directional spatial sampling of canopy gap than LAI-2000 instrument, which relies on only five zenith angles. This allows applying Lang and Xiang (1986) method to small cells (cf. Section 2.3) for which the random distribution of foliage elements is probably more valid than over LAI-2000 rings. Besides, once the clumping index is computed for each zenith angle it is used in the modified Poisson model to compute PAI, which is theoretically more accurate than directly averaging the logarithm of gap fraction (Method 1). Note that clumping correction could have also been applied to LAI-2000 and AccuPAR measurements. But, agreement between $\mathrm{PAI}_{\text {eff }}$ retrieval techniques must be first investigated (cf. objective of this paper) before comparing PAI retrieval.

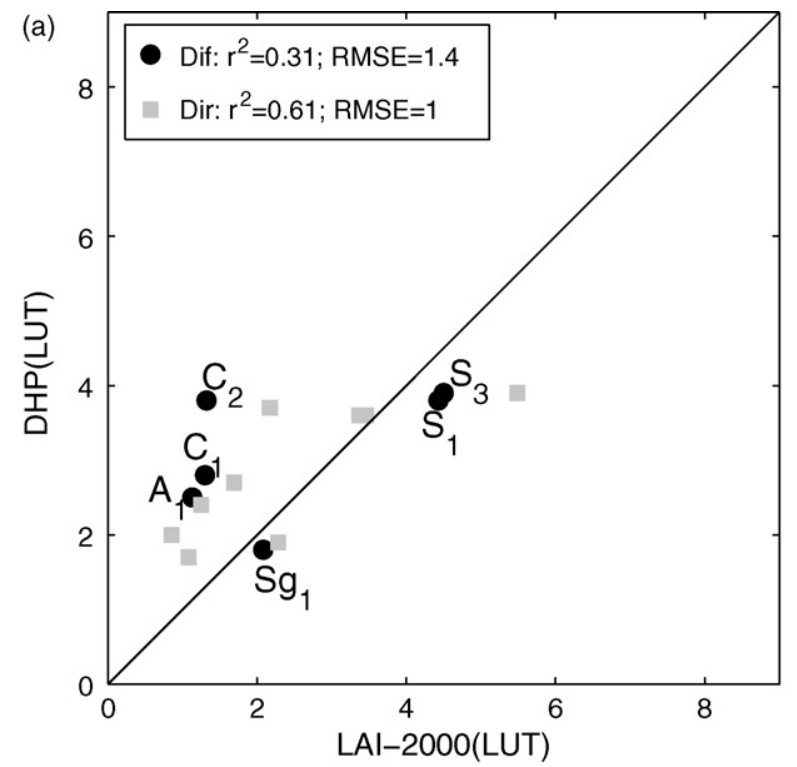

4.1.3.2. Sensitivity to gap fraction inversion methods. Fig. $6 \mathrm{~b}$ compares retrievals based on multiple directional gap fraction measurements using the LUT method and those using gap fraction measurements at the single $\theta=57.5^{\circ}$ view zenith angle (Wilson method). It indicates a very good agreement of retrievals by these two methods (RMSE 4-5\%). Disagreement between these two methods is much larger in the case of LAI2000 measurements (RMSE 35-40\%, cf. Table 1), showing the robustness of the DHP technique to the type of inversion method used to estimate $\mathrm{PAI}_{\text {eff. }}$.

4.1.3.3. Sensitivity to illumination conditions. Fig. 6c indicates that $\mathrm{PAI}_{\text {eff }}$ estimated under direct illumination are slightly lower than those retrieved under diffuse illumination. The negative bias of DHP retrievals under direct illumination may

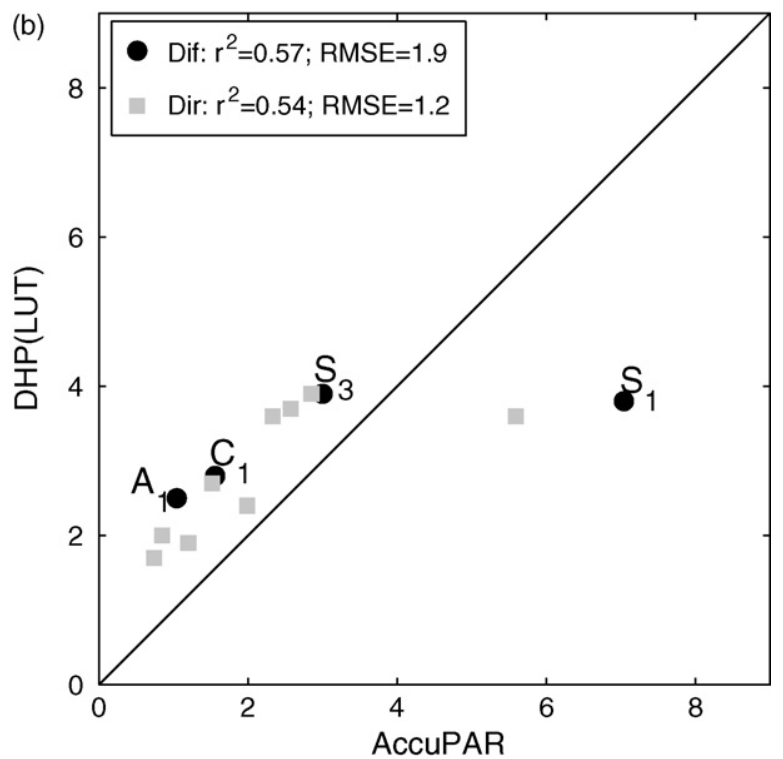

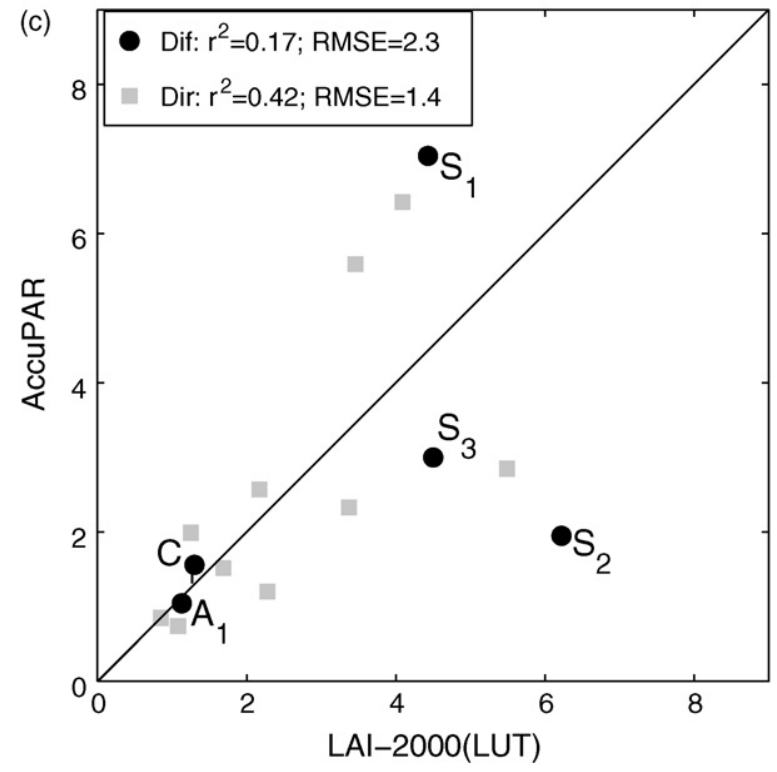

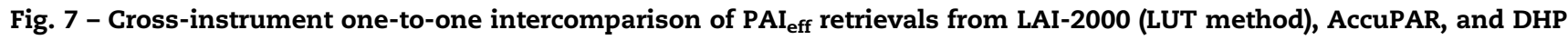
(LUT method) instruments under direct (grey square) and diffuse (black circle) illumination conditions. Each point represents an estimate at the ESU scale (obtained by averaging Method 2, cf. Section 3). The letters A, C, S, Sg stand for alfalfa, corn, soybean and sorghum. 
be due to the following optical effects. In the case of upwardlooking imagery, overexposed sunlit leaves can be indistinguishable from sky. In the case of downward-looking imagery, it can be impossible to differentiate shaded green leaves from dark or shaded soil background. In both cases, the amount of pixels with green leaves and thus $\mathrm{PAI}_{\mathrm{eff}}$ tend to be underestimated compared to the diffuse illumination case. Fig. $6 \mathrm{~d}$ shows that this sensitivity to illumination conditions is more important for downward-pointing imagery, for which diffuse conditions are recommended.

However, the magnitude of sensitivity of DHP retrievals to illumination conditions is much smaller (RMSE 14\%) than that of others instruments (RMSE 27\% for LAI-2000 and $86 \%$ for AccuPAR), suggesting that DHP is preferable among other techniques to estimate $\mathrm{PAI}_{\text {eff }}$ under varying illumination conditions. Besides, in contrast to LAI-2000, Wilson, and LUT methods show similar sensitivity to illumination conditions (results not presented here for sake of concision).

Note, also, that the sensitivity of DHP retrievals to illumination conditions over the crops under study is smaller than that generally observed over forests (Chen et al., 1991). This is probably due to the smaller size of foliage elements of forest canopy compared to those of crop canopy, making their discrimination more difficult under direct sunlight.

4.1.3.4. Sensitivity to scaling methods. Fig. $6 \mathrm{~d}$ compares $\mathrm{PAI}_{\text {eff }}$ estimates at the ESU scale using scaling Methods 1 and 2. Note that retrievals from the Wilson method are used here since LUT retrievals were not available at the point scale (cf. Section 2.3). Discrepancies between methods (RMSE 3-5\%) are much lower than those observed for LAI-2000 (Wilson method: RMSE 14-15\%) while both techniques relies on the same algorithm. This implies that the LAI-2000 gap fraction point measurements are more variable than the DHP ones. Indeed, downward-looking photographs over short canopy provide more stable measurements than those from LAI-2000, which may be disturbed by the short distance between leaves and instrument. Besides, the larger field of view of the DHP fish-eye as well as its larger azimuthal range compared to those of LAI2000 provide a better spatial sampling of transmittance, capturing most canopy spatial heterogeneity at the point scale.

\subsection{Cross-sensor intercomparison}

4.2.1. Intercomparison of DHP, LAI-2000 and AccuPAR PAI eff retrievals

We now intercompare $\mathrm{PAI}_{\text {eff }}$ estimated from LAI-2000, AccuPAR, and DHP at the ESU scale (Fig. 7). For sake of consistency in the comparison, we use the same scaling Method 2 (cf. Section 3) of transmittance over the ESU for all the instruments. For LAI-2000, we use retrievals from the LUT method which is similar to the baseline retrieval implemented in Can-Eye.

Fig. 7 shows much larger $\mathrm{PAI}_{\text {eff }}$ discrepancies between optical techniques than $\mathrm{PAI}_{\text {eff }}$ variations in sensitivity tests (cf. Table 2), suggesting that method and parameter uncertainties can only partially explain inter-sensor $\mathrm{PAI}_{\text {eff }}$ discrepancies. While RMSE is slightly lower for direct illuminations, the overall agreement does not significantly depend on illumination conditions. The following analysis will be thus focused on measurements under diffuse illumination, which is expected to be optimal for all instruments. Differences in instrument footprint, zenith angle spatial resolution (coarser for LAI-2000 than DHP imagery), and azimuthal range (AccuPAR integrates radiation over the entire field of view while LAI-2000 uses 0-270 azimuthal range, and DHP azimuthal range varies with the surface of masked pixel) lead to different spatial sampling of transmittance by each instrument, resulting in different estimated $\mathrm{PAI}_{\text {eff }}$ values over heterogeneous canopies (e.g. corn ESU, see labels C2 and C1 in Fig. 7). However, these differences should be reduced at the ESU scale and are not sufficient to explain the important $\mathrm{PAI}_{\text {eff }}$ discrepancies shown in Fig. 7. In the following, we interpret part of $\mathrm{PAI}_{\text {eff }}$ discrepancies between instruments in terms of crop properties (e.g. canopy height; presence or lack of senescent vegetation).

We first compare DHP versus AccuPAR and LAI-2000 retrievals. On average, for most ESUs, $\mathrm{PAI}_{\text {eff }}$ values from DHP are higher than those from LAI-2000 and AccuPAR by 1.08

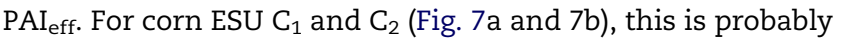
due to the presence of senescent leaves whose transmittance may be larger than that of green leaves, resulting in lower $\mathrm{PAI}_{\text {eff }}$ estimates from both LAI-2000 and AccuPAR. Indeed, the assumption of black foliage for LAI-2000 as well as the default absorptivity coefficient $(a=0.9)$ for AccuPAR may not be appropriate for thin senescent leaves. Conversely, in the case of DHP baseline method, based on RGB classification into two classes (vegetation versus background), senescent leaves are classified by the operator into the same vegetation class than green leaves. Thus, compared to AccuPAR and LAI-2000, gap fraction measurements and $\mathrm{PAI}_{\text {eff }}$ estimates from DHP technique should be less affected by possible higher transmittance of senescent leaves. Large discrepancies are also observed over short canopies (soybean: $S_{1}, S_{3}$ and alfalfa: $A_{1}$, cf. Fig. 7a and 7b). LAI-2000 and AccuPAR devices used below short canopies were probably too close to the leaves to be able to properly capture canopy gap spatial distribution. This can lead to local artifacts such as a big leaf masking the whole field-of-view of the instrument, resulting in transmittance measurements close to zero (saturation domain) and thus large $\mathrm{PAI}_{\text {eff }}$ values (e.g. $\mathrm{S}_{1}$ for AccuPAR). Besides, instruments placed below short canopies may disturb canopy structure, affecting measurements. Conversely, downward-looking DHPs over short canopies are not affected by these artifacts, resulting in more realistic $\mathrm{PAI}_{\text {eff }}$ estimates. Better agreement between retrieval techniques was found for tall canopy without senescent vegetation (e.g. $\mathrm{Sg}_{1}$ under diffuse conditions, cf. Fig. 7a).

Comparison of LAI-2000 and AccuPAR retrievals indicate good agreement for $\mathrm{PAI}_{\text {eff }}$ lower than 3 (on average, difference of $0.38 \mathrm{PAI}_{\text {eff }}$, cf. Fig. 7c). Discrepancies substantially increase at larger $\mathrm{PAI}_{\mathrm{eff}}$ (on average, difference of $2.35 \mathrm{PAI}_{\text {eff }}$ ). Maximum differences are obtained for ESU $\mathrm{S}_{1}\left(2.6 \mathrm{PAI}_{\text {eff }}\right)$ and $\mathrm{S}_{2}\left(4.3 \mathrm{PAI}_{\text {eff }}\right)$ for which the short canopy affects measurements, and was thus not optimal to properly compare retrievals from LAI-2000 and AccuPAR. Other sources of discrepancies between LAI-2000 and AccuPAR retrievals include difference in azimuthal range, instrument footprint, and sensitivity to senescent vegetation. 


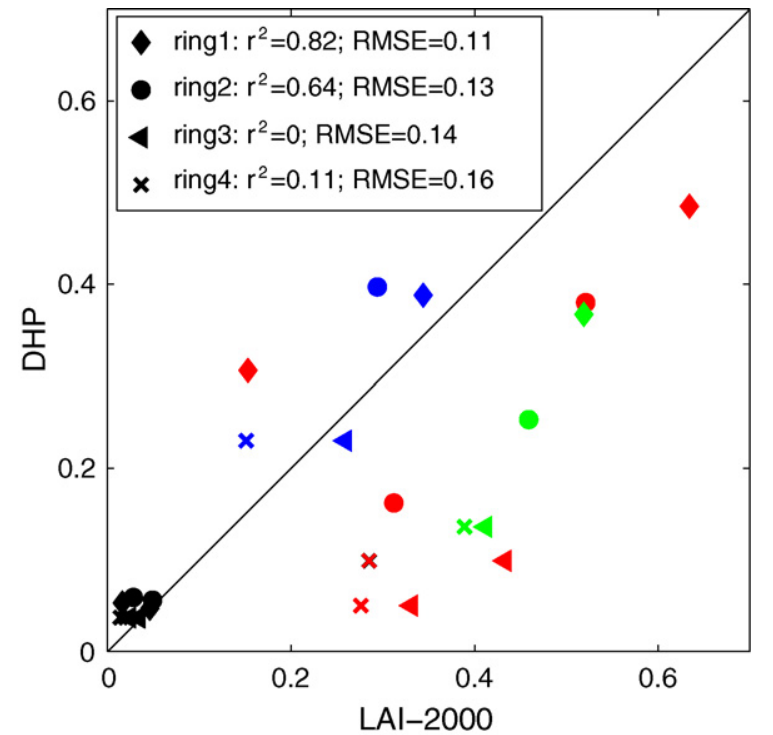

Fig. 8 - Comparison of gap fraction measurements (average value over the ESU) from LAI-2000 to those from DHP, acquired under diffuse illuminations. Blue, red, green, and black plots represent sorghum, corn, alfalfa, and soybean crops, respectively.

\subsubsection{Comparison of LAI-2000 and DHP measurements} We now further investigate differences between LAI-2000 and DHP through comparison (under diffuse illumination) of gap fraction measured by each instruments and effective Average Leaf Inclination Angle $\left(\mathrm{ALIA}_{\text {eff }}\right.$ ) estimated along with $\mathrm{PAI}_{\text {eff }}$ in each retrieval algorithm.

4.2.2.1. Gap fraction analysis. Since DHP and LAI-2000 retrieval algorithm are based on the same LUT inversion technique,

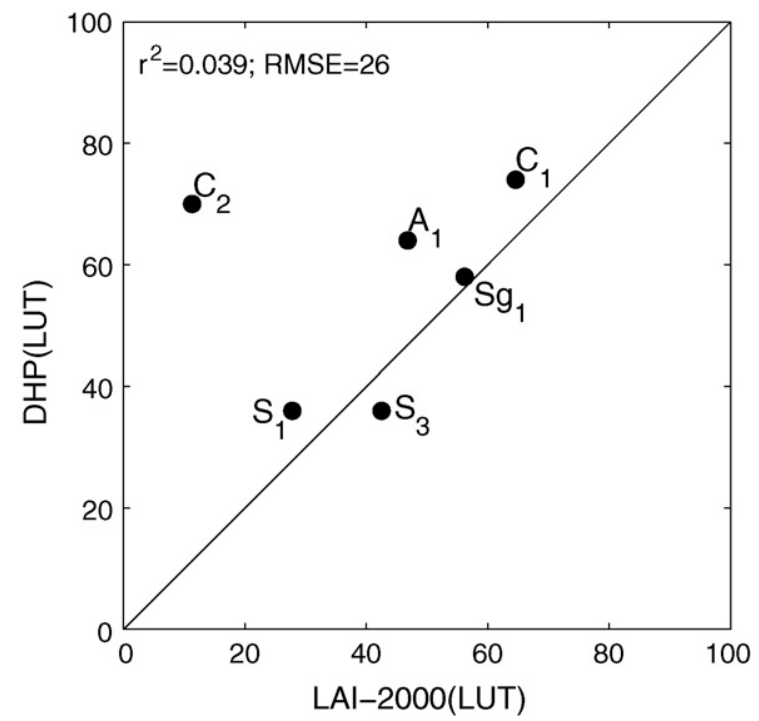

Fig. 9 - Intercomparison of ALIA eff retrievals from LAI-2000 (LUT method) and DHP (LUT method), derived under diffuse illumination conditions. Each point represents an estimate at the ESU scale. discrepancies between their PAI $_{\text {eff }}$ estimates are probably due to differences in gap fraction measurements. To compare gap fraction measurements from LAI-2000 to those from DHP, DHP gap fraction measurements are averaged over the zenith angular range of each LAI-2000 ring, assuming that simple averaging is a reasonable approximation of the angular response of the LAI-2000 detectors (LI-COR, 1991). The comparison for the fifth ring was not possible since Can-Eye does not provide any measurements for zenith angle larger than $60^{\circ}$. The analysis is performed at the ESU scale under diffuse illuminations. Discrepancies between DHP and LAI2000 gap fraction measurements yield a RMSE in between 0.1 and 0.16 (Fig. 8) and reflect the differences in $\mathrm{PAI}_{\text {eff }}$ values shown in Fig. 7a. Best agreement is found for tall canopy with low heterogeneity (Sorghum). The large discrepancies observed over heterogeneous (Corn) and short canopies (alfalfa, soybean) reflect substantial differences in spatial sampling of transmittance between DHP and LAI-2000 (cf. Section 4.1.3.4). This may be caused by variations in instrument footprint (downward for DHP versus upward for LAI2000) and azimuthal range as well as by minimum height of measurements for LAI-2000 that restricts accounting for the lower leaves. Other sources of departure between LAI-2000 and DHP gap fraction measurements include classification errors in the DHP method as well as higher transmittance of senescent leaves (corn) and multiple scattering for LAI-2000.

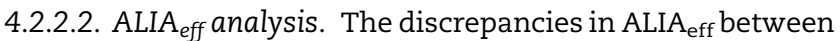
LAI-2000 and DHP under diffuse condition (Fig. 9) reflect those observed for $\mathrm{PAI}_{\text {eff. }}$ Good agreement is found over sorghum $\left(\mathrm{Sg}_{1}\right)$ showing quite realistic value (ALIA $_{\text {eff }} \sim 56^{\circ}$ for LAI-2000 and $58^{\circ}$ for DHP) for spherical leaf angle distribution canopy.

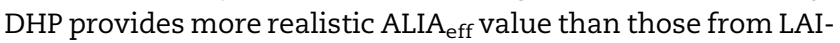
2000 for corn canopy (particularly ESU $C_{2}$ ) whose leaf angle distribution is frequently erectophylle (ALIA $>60^{\circ}$ ). Over soybean $\left(\mathrm{S}_{1}\right.$ and $\left.\mathrm{S}_{3}\right)$, DHP provides consistent ALIA $\mathrm{Aff}\left(\sim 36^{\circ}\right)$ values characterizing planophylle canopy while LAI-2000 $\mathrm{ALIA}_{\text {eff }}$ estimates are more variable $\left(28^{\circ}\right.$ for $\mathrm{S}_{1}$ and $42^{\circ}$ for $\mathrm{S}_{3}$ ), probably due to inaccurate measurements below short canopies (cf. Section 4.2.1). DHP and LAI-2000 tend to overestimate and underestimate, respectively, ALIA eff $_{\text {over alfalfa }}$ which generally has a spherical leaf angle distribution (ALIA $\sim 57^{\circ}$ ). Note, however, that evaluating the estimated ALIA $_{\text {eff }}$ is a difficult task since this parameter is not only species dependent and may vary with particular local conditions, phenological stage and sun position (e.g. soybean).

\section{Conclusion}

Relative performances of LAI-2000, AccuPAR, and DHP, with respect to retrievals of effective Plant Area Index ( $\mathrm{PAI}_{\text {eff }}$ ) over croplands were investigated here. The study focused on quantifying $\mathrm{PAI}_{\text {eff }}$ sensitivity to the type of instrument, gap fraction inversion methods, and environmental conditions (canopy heterogeneity, senescent vegetation, illumination conditions). This research was performed in support to the validation of coarse-resolution satellite data with ground measurements of $\mathrm{PAI}_{\text {eff }}$ collected according to sampling strategy over Elementary Sampling Unit ( 30 $\mathrm{m} \times 30 \mathrm{~m})$. 
Our results indicate that the sensitivity of $\mathrm{PAI}_{\mathrm{eff}}$ estimates to the several factors investigated generally increases at $\mathrm{PAI}_{\text {eff }}$ larger than three and under direct illumination. As compared to LAI-2000, DHP technique appears more robust with respect to gap fraction inversion methods used to retrieve $\mathrm{PAI}_{\text {eff }}$ (i.e., multiple versus single $\left(57.5^{\circ}\right)$ zenithal direction gap fraction measurements). AccuPAR retrieval technique relies on several key parameters, characterizing illumination conditions and leaf properties, which were shown to be an important source of $\mathrm{PAI}_{\text {eff }}$ uncertainty. Thus, AccuPAR direct use over a wide range of vegetation types and environmental conditions (as required by LAI validation activities) may be limited. LAI-2000 measurements are unstable under direct sunlight conditions and diffuse conditions should thus be selected for optimal performances in agreement with results from Literature. As sensitivity of AccuPAR estimates to retrieval parameters and measurement errors increases under direct illuminations, AccuPAR should also preferentially be used under diffuse illuminations. Overall, DHP retrievals show the lowest sensitivity to illumination conditions ( $14 \%$ versus $28 \%$ and $86 \%$ for LAI-2000 and AccuPAR, respectively), with however, better performances under diffuse illumination, particularly for downward-pointing photographs. Finally, we demonstrated that AccuPAR and DHP provide better spatial sampling of gap fraction than LAI-2000 and capture more canopy spatial heterogeneity at the point scale than LAI-2000. However, this result has to be refined over more heterogeneous canopies, with emphasis on evaluating appropriate sampling scheme and averaging method to capture canopy heterogeneity.

Intercomparison of $\mathrm{PAI}_{\text {eff }}$ from AccuPAR, DHP, and LAI2000 at the ESU scale indicates a larger magnitude of discrepancies between instruments than $\mathrm{PAI}_{\text {eff }}$ variations in the sensitivity analysis, especially at high $\mathrm{PAI}_{\text {eff. Large }}$ discrepancies between retrieval techniques are observed for short canopies over which downward-pointing DHP technique performs better than upward LAI-2000 and AccuPAR measurements. Better agreement was found for tall canopies without senescent vegetation and low spatial heterogeneity. Overall, discrepancies in $\mathrm{PAI}_{\text {eff }}$ between instruments are mainly explained by differences in spatial sampling of transmittance between instruments (over short and heterogeneous canopies) caused by variations in instrument footprint, zenith angle spatial resolution (coarser for LAI-2000 than DHP) and azimuthal range.

The discrepancies in $\mathrm{PAI}_{\text {eff }}$ estimates between optical instruments over croplands reported in this study contrast results from other studies, especially for forest, which indicate better agreement (Hyer and Goetz, 2004; Zhang et al., 2005). Note, however, that no similar intercomparison work was performed in the past over croplands to establish a reference. Besides, former studies relied on different image processing approaches to derive gap fraction from DHP imagery. Overall, our results serve as an indication of the upper limit of inconsistencies between optical measurements over croplands, due to fairly extreme, but not uncommon in practice, conditions for instrument utilization (i.e., presence of senescent vegetation and short canopies). Still, our study brings insights about instrument performances and limits in the context of validation protocols, and helps identifying repeatable and stable measurement of canopy properties. Accord- ing to this work, DHP is the most robust optical technique in terms of low sensitivity to illumination conditions, its ability to capture gap fraction over short canopy (using downwardlooking photograph) and independence from ancillary information on canopy optical properties. Besides, it provides a far finer directional spatial sampling of canopy gaps than other instruments, allowing to deriving a clumping index along multiple zenith angles. DHP technique can thus be used through the day to reduce the field campaign length, and can easily be applied to a large range of canopy structures and environmental conditions as required by validation protocols.

Further research is required to refine this analysis. To assess the lower limit of inconsistencies between instruments, the retrievals should be compared under "optimal" environmental conditions, namely green vegetation, large fields, and diffuse illumination. The intercomparison should be performed over a range of vegetation structures, phenological stages, soil properties, topography features, and environmental conditions. The performance of the DHP technique should be further assessed as a function of different algorithms, namely compare retrievals by CanEye algorithm relying on RGB color classification of the image and by Leblanc et al. (2005) algorithm relying on brightness thresholds in the blue band of the image. New DHP methods have also to be tested to separate non-green elements from green foliage, possibly using near-infrared imagery that should be more appropriate than standard visible bands. Finally, further work should involve destructive measurements to assess absolute uncertainty of each optical technique. This information is currently lacking for most validation datasets, and is critical to properly validate remote sensing LAI products.

\section{Acknowledgements}

This study was funded by NASA's Terrestrial Ecology Program (D. Wickland, program manager). We thank researchers at INTA-Manfredi, Argentina, especially Mr. Juan E. Pappalardo, for proving access to the Cordoba site, and help during the field experiment. We also thank Dr. Marie Weiss at INRA, UMR 1114 EMMAH, Avignon, France for providing support with Look-Up Table retrieval technique for processing LAI-2000 measurements, Can-Eye software for processing DHP images, and general comments.

\section{REFERENCES}

Barclay, H.J., Trofymow, J.A., Leach, R.I., 2000. Assessing bias from boles in calculating leaf area index in immature Douglas-fir with the LI-COR canopy analyzer. Agricultural and Forest Meteorology 100 (2-3), 255-260.

Baret, F., Weiss, M., Allard, D., Garrigues, S., Leroy, M., Jeanjean, H., Fernandes, R., Myneni, R.B., Morissette, J.T., Privette, J., Bohbot, H., Bosseno, R., Dedieu, G., Di Bella, C., Espana, M., Gond, V., Gu, X.F., Guyon, D., Lelong, C., Maisongrande, P., Mougin, E., Nilson, T., Veroustraete, F., Vintilla, R. VALERI: a network of sites and a methodology for the validation of medium spatial resolution land satellite product. Remote Sensing of Environment, submitted for publication. 
Begue, A., 1993. Leaf area index, intercepted photosynthetically active radiation, and spectral vegetation indexes-a sensitivity analysis for regular-clumped canopies. Remote Sensing of Environment 46 (1), 45-59.

Breda, N.J.J., 2003. Ground-based measurements of leaf area index: a review of methods, instruments and current controversies. Journal of Experimental Botany 54 (392), 2403-2417.

Campbell, G.S., 1986. Extinction coefficients for radiation in plant canopies calculated using an ellipsoidal inclination angle distribution. Agricultural and Forest Meteorology 36, 317-321.

Campbell, G.S., Norman, J.M., 1998. An Introduction to Environmental Biophysics. Springer-Verlag, New York, $286 \mathrm{pp}$.

Chapman, L., 2007. Potential applications of near infarared hemispherical imagery in forest environments. Agricultural and Forest Meteorology 143, 151-156.

Chen, J.M., Black, T.A., 1992. Defining leaf area index for non-flat leaves. Plant Cell Environment 15, 421-429.

Chen, J.M., Black, T.A., Adams, R.S., 1991. Evaluation of hemispherical photography for determining plant area index and geometry of a forest stand. Agricultural and Forest Meteorology 56, 129-143.

Chen, J.M., Cihlar, J., 1995. Plant canopy gap-size analysis theory for improving optical measurements of leaf area index. Applied Optics 34, 6211-6222.

Chen, J.M., Rich, P., Gower, S.T., Norman, J.M., Plummer, S., 1997. Leaf area index of boreal forests: theory, techniques and measurements. Journal of Geophysical Research 102 (D24), 29429-29443.

Cohen, S., Rao, R.S., Cohen, Y., 1997. Canopy transmittance inversion using a line quantum probe for a row crop. Agricultural and Forest Meteorology $86(3-4)$, 225-234.

Combal, B., Baret, F., Weiss, M., Trubuil, A., Macé, D., Pragnère, A., Myneni, R., Knyazikhin, Y., Wang, L., 2002. Retrieval of canopy biophysical variables from bi-directional reflectance data. Using prior information to solve the ill-posed inverse problem. Remote Sensing of Environment 84, 1-15.

Decagon Devices Inc, 2001. Decagon AccuPar Ceptometer Operating Manual. Decagon Devices Inc., Pullman, WA, $142 \mathrm{pp}$.

Fernandes, R.A., Butson, C., Leblanc, S., Latifovic, R., 2003. A, Landsat-5 TM and Landsat-7ETM+ based accuracy assessment of leaf area index products for Canada derived from SPOT4/VGT data. Canadian Journal of Remote Sensing 29 (2), 241-258.

Frazer, G.W., Fournier, R.A., Trofymow, J.A., Hall, J.R., 2001. A comparison of digital and film fisheye photography for analysis of forest canopy structure and gap light transmission. Agricultural and Forest Meteorology 109, 249-263.

Garrigues, S., Allard, D., Baret, F., 2006. Influence of the spatial heterogeneity on the non linear estimation of Leaf Area Index from moderate resolution remote sensing data. Remote Sensing of Environment 106, 286-298.

Garrigues, S., Lacaze, R., Baret, F., Morisette, J.T., Weiss, M., Nickeson, J., Fernandes, R., Plummer, S., Shabanov, N.V., Myneni, R., Knyazikhin,Y., Yang, W., (2008). Validation and inter-comparison of global Leaf Area Index product derived from remote sensing data. Journal of Geophysical Research, in press.

Gower, S.T., Kucharik, C.J., Norman, J.M., 1999. Direct and indirect estimation of leaf area index, FAPAR, and net primary production of terrestrial ecosystems. Remote Sensing of Environment 70 (1), 29-51.

Hyer, E.J., Goetz, S.J., 2004. Comparison and sensitivity analysis of instruments and radiometric methods for LAI estimation: assessments from a boreal forests site. Agricultural and Forest Meteorology 122, 157-174.

Jonckheere, I., Fleck, S., Nackaerts, K., Muys, B., Coppin, P., Weiss, M., Baret, F., 2004. Review of methods for in situ leaf area index determination: Part I. Theories, sensors and hemispherical photography. Agricultural and Forest Meteorology 121 (1-2), 19-35.

Kucharik, C.J., Norman, J.M., Gower, S.T., 1998. Measurements of branch area and adjusting leaf area index indirect measurements. Agricultural and Forest Meteorology 91 (12), 69-88.

Lang, A.R.G., Xiang, Y., 1986. Estimation of leaf area index from transmission of direct sunlight in discontinuous canopies. Agricultural and Forest Meteorology 35, 229-243.

Leblanc, S.G., Chen, J., Fernandes, R., Deering, D.W., Conley, A., 2005. Methodology comparison for canopy structure parameter extraction from digital hemispherical photography in boreal forests. Agricultural and Forest Meteorology 129, 187-207.

Leblanc, S.G., Chen, J.M., 2001. A practical scheme for correcting multiple scattering effects on optical LAI measurements. Agricultural and Forest Meteorology 110 (2), 125-139.

LI-COR (1991), Plant Canopy Analyser Operating Manual. Lincoln, NE: LI-COR Inc. (90 p).

Martens, S.N., Ustin, S.L., Rousseau, R.A., 1993. Estimation of tree canopy Leaf-Area Index by gap fraction analysis. Forest Ecology and Management 61, 91-108.

Miller, J.B., 1967. A formula for average foliage density. Australian Journal of Botany 15, 141-144.

Morisette, J.T., Baret, F., Privette, J.L., Myneni, R.B., Nickeson, J., Garrigues, S., Shabanov, N.V., Weiss, M., Fernandes, R., Leblanc, S., Kalacska, M., Sánchez-Azofeifa, G.A., Chubey, M., Rivard, B., Stenberg, P., Rautiainen, M., Voipio, P., Manninen, T., Pilant, A., Lewis, T., liames, J., Colombo, R., Meroni, M., Busetto, L., Cohen, W., Turner, D., Warner, E.D., Petersen, G.W., Seufert, G., Cook, R., 2006. Validation of global moderate-resolution LAI Products: a framework proposed within the CEOS Land Product Validation subgroup. IEEE Transactions on Geoscience and Remote Sensing 44, 1804-1814.

Nilson, T., 1971. A theoretical analysis of the frequency gaps in plant stands. Agricultural and Forest Meteorology 8, 25-28.

Norman, J.M., Jarvis, P.G., 1975. Photosynthesis in sitka spruce (Picea Sitchensis (Bong) Carr.): V. Radiation penetration and a test case. Journal of Applied Ecology 12 (3), 839-878.

Planchais, I., Pontailler, J.-Y., 1999. Validity of leaf areas and angles estimated in a beech forest from analysis of gap frequencies, using hemispherical photographs and a plant canopy analyser. Annals Forest Science 56, 1-10.

Stenberg, P., 1996. Correcting LAI2000 estimates for the clumping of needles in shoots of conifers. Agricultural and Forest Meteorology 43, 20-34.

Stenberg, P., Nilson, T., Smolander, S., Voipio, P., 2003. Gap fraction based estimation of LAI in scots pine stands subjected to experimental removal of branches and stems. Canadian Journal of Remote Sensing 29, 363-370.

Tan, B., Hu, J., Zhang, P., Huang, D., Shabanov, N.V., Weiss, M., Knyazikhin, Y., Myneni, R.B., 2005. Validation of MODIS LAI product in croplands of Alpilles, France. Journal of Geophysical Research 110, D01107, doi:10.1029/ 2004JD004860.

Van Gardingen, P.R., Jackson, G.E., Hernandez-Daumas, S., Russell, G., Sharp, L., 1999. Leaf Area Index estimates obtained for clumped canopies using hemispherical photography. Agricultural and Forest Meteorology 94 (3-4), 243-257.

Weiss, M., Baret, F., Garrigues, S., Lacaze, R., 2007. LAI and fAPAR CYCLOPES global products derived from VEGETATION. Part 2: Validation and intercomparison with 
MODIS Collection 4 products. Remote Sensing of Environment 110 (3), 317-331.

Weiss, M., Baret, F., Smith, G.J., Jonckheere, I., Coppin, P., 2004. Review of methods for in situ leaf area index (LAI) determination: Part II. Estimation of LAI, errors and sampling. Agricultural and Forest Meteorology 121 (1-2), 37-53.

Welles, J.M., 1990. Some indirect methods of estimating canopy structure. Remote Sensing Reviews 5, 31-43.

Welles, J.M., Norman, J.M., 1991. Instrument for indirect measurement of canopy architecture. Agronomy Journal 83 (5), 818-825.

White, M., Asner, G.P., Nemani, R.R., Privette, J.L., Running, S.W. 2000. Measuring fractional cover and leaf area index in arid ecosystems: digital camera, radiation transmittance, and laser altimetry methods. Remote Sensing of Environment 74, 45-57.

Wilhelm, W.W., Ruwe, K., Schlemmer, M.R., 2000. Comparison of the three Leaf Area Index meters in a corn canopy. Crop Science 40.

Wilson, J.W., 1963. Estimation of foliage denseness and foliage angle by inclined point quadrats. Australian Journal of Botany 11, 95-105.

Zhang, Y., Chen, J.M., Miller, J.R., 2005. Determining digital hemispherical photograph exposure for leaf area index estimation. Agricultural and Forest Meteorology 133 (1-4), 166-181. 